\title{
AN ORE-TYPE THEOREM FOR PERFECT PACKINGS IN GRAPHS
}

\author{
DANIELA KÜHN, DERYK OSTHUS AND ANDREW TREGLOWN
}

\begin{abstract}
We say that a graph $G$ has a perfect $H$-packing (also called an $H$-factor) if there exists a set of disjoint copies of $H$ in $G$ which together cover all the vertices of $G$. Given a graph $H$, we determine, asymptotically, the Ore-type degree condition which ensures that a graph $G$ has a perfect $H$-packing. More precisely, let $\delta_{\text {Ore }}(H, n)$ be the smallest number $k$ such that every graph $G$ whose order $n$ is divisible by $|H|$ and with $d(x)+d(y) \geq k$ for all non-adjacent $x \neq y \in V(G)$ contains a perfect $H$-packing. We determine $\lim _{n \rightarrow \infty} \delta_{\text {Ore }}(H, n) / n$.
\end{abstract}

\section{INTRODUCTION}

1.1. Perfect packings in graphs of large minimum degree. Given two graphs $H$ and $G$, an $H$-packing in $G$ is a collection of vertex-disjoint copies of $H$ in $G$. An $H$-packing is called perfect if it covers all the vertices of $G$. In this case one also says that $G$ contains an $H$-factor. $H$-packings are generalisations of graph matchings (which correspond to the case when $H$ is a single edge).

In the case when $H$ is an edge, Tutte's theorem characterises those graphs which have a perfect $H$-packing. However, for other connected graphs $H$ no characterisation is known. Furthermore, Hell and Kirkpatrick [4 showed that the decision problem whether a graph $G$ has a perfect $H$-packing is NP-complete precisely when $H$ has a component consisting of at least 3 vertices. It is natural therefore to ask for simple sufficient conditions which ensure the existence of a perfect $H$-packing. One such result is a theorem of Hajnal and Szemerédi [3] which states that a graph $G$ whose order $n$ is divisible by $r$ has a perfect $K_{r}$-packing provided that $\delta(G) \geq(1-1 / r) n$. It is easy to see that the minimum degree condition here is best possible. So for $H=K_{r}$, the parameter which governs the existence of a perfect $H$-packing in a graph $G$ of large minimum degree is $\chi(H)=r$.

The first two authors [13, 14] showed that for any graph $H$ either the so-called critical chromatic number or the chromatic number of $H$ is the relevant parameter. Here the critical chromatic number $\chi_{c r}(H)$ of a graph $H$ is defined as

$$
\chi_{c r}(H):=(\chi(H)-1) \frac{|H|}{|H|-\sigma(H)},
$$

where $\sigma(H)$ denotes the size of the smallest possible colour class in any $\chi(H)$-colouring of $H$. Throughout the paper, we only consider graphs $H$ which contain at least one edge (without mentioning this explicitly), so $\chi_{c r}(H)$ is well defined. Note that $\chi(H)-1<$

Date: June 2, 2009.

The authors were supported by the EPSRC, grant no. EP/F008406/1. 
$\chi_{c r}(H) \leq \chi(H)$ for all graphs $H$, and $\chi_{c r}(H)=\chi(H)$ precisely when every $\chi(H)$ colouring of $H$ has colour classes of equal size. The characterisation of when $\chi(H)$ or $\chi_{c r}(H)$ is the relevant parameter depends on the so-called highest common factor of $H$, which is defined as follows.

We say that a colouring of $H$ is optimal if it uses exactly $\chi(H)=: r$ colours. Given an optimal colouring $c$ of $H$, let $x_{1} \leq x_{2} \leq \cdots \leq x_{r}$ denote the sizes of the colour classes of $c$. We write $\mathcal{D}(c):=\left\{x_{i+1}-x_{i} \mid i=1, \ldots, r-1\right\}$, and let $\mathcal{D}(H)$ denote the union of all the sets $\mathcal{D}(c)$ taken over all optimal colourings $c$ of $H$. We denote by hcf $\chi(H)$ the highest common factor of all integers in $\mathcal{D}(H)$. If $\mathcal{D}(H)=\{0\}$ then we define hcf $\mathrm{f}_{\chi}(H):=\infty$. We write $\operatorname{hcf}_{c}(H)$ for the highest common factor of all the orders of components of $H$.

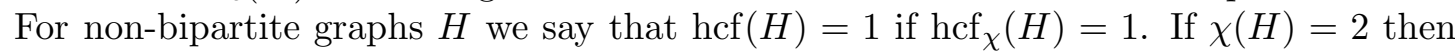
we say $\operatorname{hcf}(H)=1$ if $\operatorname{hcf}_{c}(H)=1$ and $\operatorname{hcf}_{\chi}(H) \leq 2$. (See [14] for some examples.) Put

$$
\chi^{*}(H):= \begin{cases}\chi_{c r}(H) & \text { if } \operatorname{hcf}(H)=1 ; \\ \chi(H) & \text { otherwise. }\end{cases}
$$

Also let $\delta(H, n)$ denote the smallest integer $k$ such that every graph $G$ whose order $n$ is divisible by $|H|$ and with $\delta(G) \geq k$ contains a perfect $H$-packing.

Theorem 1. 14] For every graph $H$ there exists a constant $C=C(H)$ such that

$$
\left(1-\frac{1}{\chi^{*}(H)}\right) n-1 \leq \delta(H, n) \leq\left(1-\frac{1}{\chi^{*}(H)}\right) n+C .
$$

Theorem 1 improved previous bounds by Alon and Yuster [1, who showed that $\delta(H, n) \leq(1-1 / \chi(H)) n+o(n)$, and by Komlós, Sárközy and Szemerédi [11], who replaced the $o(n)$-term by a constant depending only on $H$. Further related results are discussed in the surveys [7, [8, 12, 15, 21].

1.2. Ore-type degree conditions for perfect packings. Of course, one can also consider other types of degree conditions that ensure a perfect $H$-packing in a graph $G$. One natural such condition is an Ore-type degree condition requiring a lower bound on the sum of the degrees of non-adjacent vertices of $G$. (The name comes from Ore's theorem [16], which states that a graph $G$ of order $n \geq 3$ contains a Hamilton cycle if $d(x)+d(y) \geq n$ for all non-adjacent $x \neq y \in V(G)$.)

A result of Kierstead and Kostochka [6] on equitable colourings implies that a graph $G$ whose order $n$ is divisible by $r$ and with $d(x)+d(y) \geq 2(1-1 / r) n-1$ for all non-adjacent $x \neq y \in V(G)$ contains a perfect $K_{r}$-packing. Note that this is a strengthening of the Hajnal-Szemerédi theorem. Kawarabayashi [5] asked for the Ore-type condition which guarantees a $K_{4}^{-}$-packing in a graph $G$ covering a given number of vertices of $G$. (Here $K_{4}^{-}$denotes the graph obtained from $K_{4}$ by removing an edge.) Similarly it is natural to seek an Ore-type analogue of Theorem 1. This will be the main result of this paper (but with an $o(n)$-error term). Perhaps surprisingly, the Ore-type condition needed is not 'twice the minimum degree condition'. For some graphs $H$ it depends on the so-called colour extension number of $H$, which we will define now. Roughly speaking, this is a measure of how many extra colours we need to properly colour $H$ if we try to build this colouring by extending an $(r-2)$-colouring of a neighbourhood of a vertex of $H$. 
More precisely, suppose that $H$ is a graph with $\chi(H)=: r$ which contains a vertex $x$ for which the subgraph $H[N(x)]$ induced by the neighbourhood of $x$ is $(r-2)$-colourable. Given such a vertex $x \in V(H)$, let $m_{x}$ denote the smallest integer for which there exists an $(r-2)$-colouring of $H[N(x)]$ that can be extended to an $\left(r+m_{x}\right)$-colouring of $H$. The colour extension number $C E(H)$ of $H$ is defined as

$$
C E(H):=\min \left\{m_{x} \mid x \in V(H) \text { with } \chi(H[N(x)]) \leq r-2\right\} .
$$

If $\chi(H[N(x)])=r-1$ for all $x \in V(H)$ we define $C E(H):=\infty$. So every bipartite graph $H$ without isolated vertices has $C E(H)=\infty$. All other bipartite graphs $H$ have $C E(H)=0$. In general, $1 \leq C E(H)<\infty$ if for any optimal colouring of $H$ and any $v \in V(H), N(v)$ lies in exactly $r-1$ colour classes of $H$, but there exists a vertex $x \in V(H)$ such that $\chi(H[N(x)]) \leq r-2$. Note that in this case $C E(H) \leq r-2$. (Indeed, we can colour $H-N(x)$ with $r$ different colours to obtain a $(2 r-2)$-colouring of $H$.)

In order to help the readers to familiarize themselves with the notion of the colour extension number we now give a number of examples. $\chi\left(K_{4}^{-}\right)=3$ and $\chi\left(K_{4}^{-}[N(x)]\right)=2$ for every vertex $x$ of $K_{4}^{-}$. Thus $C E\left(K_{4}^{-}\right)=\infty$. Next consider the graph $F^{\diamond}$ obtained from the complete 3-partite graph $K_{2,2,2}$ by removing an edge $x y$ of $K_{2,2,2}$ and adding a new vertex $z$ which is adjacent to $x$ and $y$ only. Then $\chi\left(F^{\diamond}\right)=3, \chi\left(F^{\diamond}[N(w)]\right)=2$ for every vertex $w \neq z$ in $F^{\diamond}$ and $\chi\left(F^{\diamond}[N(z)]\right)=1$. Note that in any 3-colouring of $F^{\diamond}$, $x$ and $y$ are coloured differently. So if we 1-colour $N(z)=\{x, y\}$, this colouring can be extended to a 4-colouring of $F^{\diamond}$ but not a 3-colouring. Thus $C E\left(F^{\diamond}\right)=1$.

For each $k \geq 1$ and $r \geq k+2$ we now give an example of a family of graphs $H^{\diamond}$ with $C E\left(H^{\diamond}\right)=k$ and $\chi\left(H^{\diamond}\right)=r$. Consider a complete $r$-partite graph whose vertex classes $V_{1}, \ldots, V_{r}$ have size $>k$. Let $H^{\diamond}$ be obtained from this graph by deleting the edges of $k$ vertex-disjoint copies $K^{1}, \ldots, K^{k}$ of $K_{k+1}$ which lie in $V_{1} \cup \cdots \cup V_{k+1}$, and by adding a new vertex $x$ which is adjacent to the $k(k+1)$ vertices lying in these copies of $K_{k+1}$ as well as to all the vertices in $V_{k+2}, \ldots, V_{r-1}$ (see Figure 1). Note that

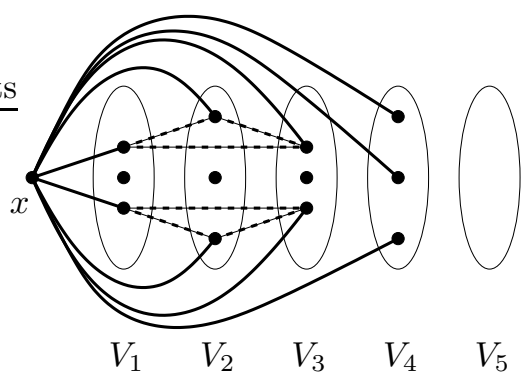

Figure 1. The graph $H^{\diamond}$ in the case when $k=2, r=5$ and when each $V_{i}$ has size 3 . The dashed lines indicate the deleted edges.

$\chi\left(H^{\diamond}\right)=r$. Furthermore, any vertex $y \in V_{1} \cup \cdots \cup V_{r}$ lies in a copy of $K_{r}$ in $H^{\diamond}$. So $\chi\left(H^{\diamond}[N(y)]\right)=r-1$. However, the subgraph $D:=H^{\diamond}\left[N(x) \cap V_{1} \cap \cdots \cap V_{k+1}\right]$ has a $k$-colouring $c_{x}^{\prime}$ with colour classes $V\left(K^{1}\right), \ldots, V\left(K^{k}\right)$ and it is easy to check that this is the only $k$-colouring of $D$ (and so in particular $\chi(D)=k$ ). Thus $\chi\left(H^{\diamond}[N(x)]\right)=r-2$ 
and the only $(r-2)$-colouring of $H^{\diamond}[N(x)]$ is the one which agrees with $c_{x}^{\prime}$ on $D$ and colours each of $V_{k+2}, \ldots, V_{r-1}$ with a new colour. Let $c_{x}$ denote this colouring. When extending $c_{x}$ to a proper colouring of $H^{\diamond}$ we cannot reuse the $r-2$ colours used in $c_{x}$ since every $y \in V\left(H^{\diamond}\right) \backslash N(x)$ is adjacent to a vertex in each colour class of $c_{x}$. As $\chi\left(H^{\diamond}-N(x)\right)=r-(r-k-2)=k+2$ this means that we require $r+k$ colours in total to extend $c_{x}$ to a proper colouring of $H^{\diamond}$. Thus $C E\left(H^{\diamond}\right)=k$.

Let

$$
\chi_{\mathrm{Ore}}(H):= \begin{cases}\chi(H) & \text { if } \operatorname{hcf}(H) \neq 1 \text { or } C E(H)=\infty ; \\ \max \left\{\chi_{c r}(H), \chi(H)-\frac{2}{C E(H)+2}\right\} & \text { otherwise. }\end{cases}
$$

Recall that $C E\left(K_{4}^{-}\right)=\infty$ and $C E\left(F^{\diamond}\right)=1$, where $F^{\diamond}$ was defined above. So $\chi_{\text {Ore }}\left(K_{4}^{-}\right)=$ $\chi\left(K_{4}^{-}\right)=3$. Any 3 -colouring of $F^{\diamond}$ has one colour class of size 3 and two colour classes of size 2. So $\operatorname{hcf}\left(F^{\diamond}\right)=1$ and thus $\chi_{\text {Ore }}\left(F^{\diamond}\right)=\max \left\{\chi_{c r}\left(F^{\diamond}\right), 3-2 / 3\right\}=\max \{14 / 5,7 / 3\}=$ $14 / 5$.

Note that if $\operatorname{hcf}(H)=1$ and $C E(H)=0$ then $\chi_{\text {Ore }}(H)=\chi_{c r}(H)$ (an odd cycle of length at least 5 provides an example of such a graph $H$ ). On the other hand, one can choose the sizes of the vertex classes $V_{i}$ in the preceding example $H^{\diamond}$ so that $\chi_{\mathrm{Ore}}\left(H^{\diamond}\right)$ lies strictly between $\chi_{c r}\left(H^{\diamond}\right)$ and $\chi\left(H^{\diamond}\right)$. (For instance, take $k$ large, $\left|V_{1}\right|=k+1$, $\left|V_{2}\right|=2 k$ and $\left|V_{i}\right|=2 k+1$ for all $i \geq 3$. Then $\chi_{c r}\left(H^{\diamond}\right)$ is close to $\chi\left(H^{\diamond}\right)-1 / 2$, $\operatorname{hcf}\left(H^{\diamond}\right)=1$ and so $\chi_{\text {Ore }}\left(H^{\diamond}\right)=\chi\left(H^{\diamond}\right)-2 /(k+2)$.)

Given a graph $H$, let $\delta_{\text {Ore }}(H, n)$ be the smallest integer $k$ such that every graph $G$ whose order $n$ is divisible by $|H|$ and with $d(x)+d(y) \geq k$ for all non-adjacent $x \neq y \in V(G)$ contains a perfect $H$-packing. Roughly speaking, our next result states that when considering an Ore-type degree condition, for any graph $H, \chi_{\text {Ore }}(H)$ is the relevant parameter which governs the existence of a perfect $H$-packing. In particular, it implies that we do not have a 'dichotomy' involving only $\chi(H)$ and $\chi_{c r}(H)$ as in Theorem 1 .

Theorem 2. For every graph $H$ and each $\eta>0$ there exists a constant $C=C(H)$ and an integer $n_{0}=n_{0}(H, \eta)$ such that if $n \geq n_{0}$ then

$$
2\left(1-\frac{1}{\chi_{\text {Ore }}(H)}\right) n-C \leq \delta_{\text {Ore }}(H, n) \leq 2\left(1-\frac{1}{\chi_{\text {Ore }}(H)}+\eta\right) n .
$$

So for example, Theorem 2 implies that $\lim _{n \rightarrow \infty} \delta_{\text {Ore }}\left(K_{4}^{-}, n\right) / n=4 / 3$ and $\lim _{n \rightarrow \infty} \delta_{\text {Ore }}\left(F^{\diamond}, n\right) / n=9 / 7$.

The upper bound in Theorem 2 follows from Lemmas 11 and 12 in Section 3 , which in turn are proved in Sections 3 and 7. The lower bound is proved in Section 2, For every graph $H$ there are infinitely many values of $n$ for which we can take $C=2$ in Theorem 2, In fact, if $\operatorname{hcf}(H) \neq 1$ or $C E(H)=\infty$ then $C=2$ suffices for all $n$ divisible by $|H|$. In general $C \leq 2|H|^{4}$ (see Section 2). It would be interesting to know whether one can replace the error term $\eta n$ by a constant depending only on $H$.

1.3. Almost perfect packings. The critical chromatic number was first introduced by Komlós [9], who showed that it is the relevant parameter when considering 'almost' perfect $H$-packings. 
Theorem 3. 9] For every graph $H$ and each $\gamma>0$ there exists an integer $n_{0}=n_{0}(\gamma, H)$ such that every graph $G$ of order $n \geq n_{0}$ and minimum degree at least $\left(1-1 / \chi_{c r}(H)\right) n$ contains an $H$-packing which covers all but at most $\gamma n$ vertices of $G$.

It is easy to see that the bound on the minimum degree in Theorem 3 is best possible. In the proof of Theorem 2 we will use the following result which provides an Oretype analogue of Theorem [3. Again, the critical chromatic number is the relevant parameter for any graph $H$. In particular, this means that Theorem 4 is a generalization of Theorem 3. The proof of Theorem 4 is almost identical to that of Theorem 3 , A sketch of the proof is given in Section 5. Full details can be found in [19].

Theorem 4. For every graph $H$ and each $\eta>0$ there exists an integer $n_{0}=n_{0}(H, \eta)$ such that if $G$ is a graph on $n \geq n_{0}$ vertices and

$$
d(x)+d(y) \geq 2\left(1-\frac{1}{\chi_{c r}(H)}\right) n
$$

for all non-adjacent $x \neq y \in V(G)$ then $G$ has an $H$-packing covering all but at most $\eta n$ vertices.

Shokoufandeh and Zhao [17] showed that in Theorem 3 the bound on the number of uncovered vertices can be reduced to a constant depending only on $H$. We conjecture that this should also be the case for Theorem 4 .

1.4. Copies of $H$ covering a given vertex. In the proof of Theorem 2 it will be useful to determine the Ore-type degree condition which guarantees a copy of $H$ covering a given vertex of $G$. Let $\delta_{\text {Ore }}^{\prime}(H, n)$ denote the smallest integer $k$ such that whenever $w$ is a vertex of a graph $G$ of order $n$ with $d(x)+d(y) \geq k$ for all non-adjacent $x \neq y \in V(G)$ then $G$ contains a copy of $H$ covering $w$. Define

$$
\chi_{\text {Ore }}^{\prime}(H):= \begin{cases}\chi(H) & \text { if } C E(H)=\infty ; \\ \chi(H)-\frac{2}{C E(H)+2} & \text { otherwise. }\end{cases}
$$

Theorem 5. For every graph $H$ and every $\eta>0$ there exists an integer $n_{0}=n_{0}(H, \eta)$ and a constant $C=C(H)$ such that if $n \geq n_{0}$ then

$$
2\left(1-\frac{1}{\chi_{\text {Ore }}^{\prime}(H)}\right) n-C \leq \delta_{\text {Ore }}^{\prime}(H, n) \leq 2\left(1-\frac{1}{\chi_{\text {Ore }}^{\prime}(H)}+\eta\right) n .
$$

Theorem 5 is proved in Section 3 , As in the case of perfect $H$-packings, the Oretype degree condition in Theorem 5 does not quite match the bound needed for the corresponding minimum degree version. Indeed, let $\delta^{\prime}(H, n)$ denote the smallest integer $k$ such that whenever $w$ is a vertex of a graph $G$ of order $n$ with $\delta(G) \geq k$ then $G$ contains a copy of $H$ covering $w$. Together with the Erdös-Stone theorem the next result implies that asymptotically $\delta^{\prime}(H, n)$ is the same as the minimum degree needed to force any copy of $H$ in a graph of order $n$.

Proposition 6. For every graph $H$ and every $\eta>0$ there exists an integer $n_{0}=n_{0}(H, \eta)$ such that if $n \geq n_{0}$ then

$$
\left(1-\frac{1}{\chi(H)-1}\right) n-1 \leq \delta^{\prime}(H, n) \leq\left(1-\frac{1}{\chi(H)-1}+\eta\right) n .
$$


The lower bound on $\delta^{\prime}(H, n)$ in Proposition 6 follows by considering a complete $(\chi(H)-1)$-partite graph $G$ whose vertex classes are as equal as possible. The proof of the upper bound is similar to Case 1 of the proof of Lemma 13 (see Section 6). Details can be found in 20].

We will not use Proposition 6 in the proof of Theorem 2, but we have included it as it helps to explain the difference between Theorems 1 and 2 . Indeed, Theorem 3 and Proposition [6] show that the minimum degree which ensures an almost perfect $H$ packing is larger than the minimum degree which guarantees a copy of $H$ covering any given vertex. In contrast, Theorems 4 and 5 imply that for some $H$ this is not true in the Ore-type case. So it is natural that $\delta_{\text {Ore }}(H, n)$ involves this property explicitly (since the property that every vertex is contained in a copy of $H$ is clearly necessary to ensure a perfect $H$-packing). In fact, this is the only real difference to the expression for $\delta(H, n)$ in Theorem 1; note that we have $\chi_{\text {Ore }}(H)=\max \left\{\chi^{*}(H), \chi_{\text {Ore }}^{\prime}(H)\right\}$ and thus Theorems 1, 2 and 5 imply that

$$
\delta_{\text {Ore }}(H, n)=\max \left\{2 \delta(H, n), \delta_{\text {Ore }}^{\prime}(H, n)\right\}+o(n) .
$$

1.5. Forcing a single copy of $H$. In view of Theorem [5, one might also wonder what Ore-type degree condition ensures at least one copy of $H$ (i.e. we do not require every vertex to lie in a copy of $H$ ). It is easy to see that if $G$ is of order $n$ then the condition is similar to the condition on the minimum degree.

Proposition 7. For every graph $H$ and every $\eta>0$ there exists an integer $n_{0}=n_{0}(H, \eta)$ such that if $n \geq n_{0}$ and $G$ is a graph on $n$ vertices which satisfies

$$
d(x)+d(y) \geq 2\left(1-\frac{1}{\chi(H)-1}+\eta\right) n
$$

for all non-adjacent $x \neq y \in V(G)$, then $G$ contains a copy of $H$.

Proposition 7 immediately follows from the Erdős-Stone theorem and the following observation (which we expect to be known, but we were unable to find a reference):

Proposition 8. Let $G$ be a graph with $d(x)+d(y) \geq 2 k$ for all non-adjacent $x \neq y \in$ $V(G)$. Then $G$ has average degree at least $k$.

To prove Proposition 8 , let $A$ be the set of vertices in $G$ whose degree is less than $k$ and let $B$ be the set of remaining vertices. Let $\bar{G}$ denote the complement of $G$ and let $F$ denote the bipartite subgraph of $\bar{G}$ induced by $A$ and $B$. Hall's theorem implies that $F$ has a matching covering all of $A$ (Hall's condition can be verified by noting that for all $X \subseteq A$ the number of edges in $F$ between $X$ and the neighbourhood of $X$ is at least $|X|(n-k-1)$ and at most $|N(X)|(n-k-1))$. Now apply the Ore-type degree condition to all pairs of vertices of $G$ which are contained in this matching.

1.6. Other structures. As mentioned earlier, packing and embedding results in graphs of large minimum degree have also been studied for other structures. It would be interesting to obtain Ore-type analogues for some of these: e.g. for the Pósa-Seymour conjecture which states that every graph $G$ on $n$ vertices with $\delta(G) \geq \frac{r}{r+1} n$ contains the $r$ th power of a Hamilton cycle. [7] contains a discussion of other Ore-type results. 


\section{Notation AND EXTREMAL EXAMPLES}

Throughout this paper we omit floors and ceilings whenever this does not affect the argument. We write $e(G)$ to denote the number of edges of a graph $G,|G|$ for its order, $\delta(G)$ and $\Delta(G)$ for its minimum and maximum degrees respectively and $\chi(G)$ for its chromatic number.

Given disjoint $A, B \subseteq V(G)$, an $A-B$ edge is an edge of $G$ with one endvertex in $A$ and the other in $B$. The number of these edges is denoted by $e_{G}(A, B)$ or $e(A, B)$ if this is unambiguous. We write $(A, B)_{G}$ for the bipartite subgraph of $G$ with vertex classes $A$ and $B$ whose edges are precisely the $A$ - $B$ edges in $G$.

Let us now prove the lower bound in Theorem 2, The next proposition deals with the case when $C E(H)=\infty$.

Proposition 9. Let $H$ be a graph with $C E(H)=\infty$. Let $n \geq|H|$. Then there exists a graph $G$ of order $n$ with

$$
d(x)+d(y) \geq 2\left(1-\frac{1}{\chi(H)}\right) n-2
$$

for all non-adjacent $x \neq y \in V(G)$ containing a vertex that does not belong to a copy of $H$. (In particular, $G$ has no perfect $H$-packing.)

Proof. Let $r:=\chi(H)$. Consider the complete $r$-partite graph of order $n$ whose vertex classes $V_{1}^{\prime}, V_{2}^{\prime}, V_{3}, \ldots, V_{r}$ have sizes as equal as possible, where $\left|V_{1}^{\prime}\right| \leq\left|V_{2}^{\prime}\right| \leq\left|V_{3}\right| \leq \cdots \leq$ $\left|V_{r}\right|$. Note that $n-\left|V_{1}^{\prime}\right|-\left|V_{2}^{\prime}\right| \geq n-2 n / r$.

Let $G$ be obtained from this graph by moving all but one vertex, $w$ say, from $V_{1}^{\prime}$ to $V_{2}^{\prime}$, by making the set $V_{2} \supseteq V_{2}^{\prime}$ thus obtained from $V_{2}^{\prime}$ into a clique and by deleting all the edges between $w$ and the vertices in $V_{2}$.

Any vertex $y \in V_{3} \cup \cdots \cup V_{r}$ satisfies $d(y) \geq n-\left\lceil\frac{n}{r}\right\rceil \geq(1-1 / \chi(H)) n-1$. Thus $d\left(y_{1}\right)+d\left(y_{2}\right) \geq 2(1-1 / \chi(H)) n-2$ for all non-adjacent $y_{1} \neq y_{2} \in V(G) \backslash\left(\{w\} \cup V_{2}\right)$. Moreover, $d(w)=n-\left|V_{1}^{\prime}\right|-\left|V_{2}^{\prime}\right| \geq n-2 n / r$ and for any $z \in V_{2}$ we have $d(z)=n-2$. So $d(w)+d(z) \geq 2(1-1 / \chi(H)) n-2$. Hence $G$ satisfies our Ore-type degree condition.

The neighbourhood of $w$ in $G$ induces an $(r-2)$-partite subgraph of $G$. Therefore, since $\chi(H[N(x)])=r-1$ for all $x \in V(H), w$ cannot play the role of any vertex in $H$. So $G$ does not contain a copy of $H$ covering $w$.

The following proposition will be used for the case when $H$ is non-bipartite and $C E(H)<\infty$.

Proposition 10. Let $H$ be a graph with $r:=\chi(H) \geq 3$ for which $m:=C E(H)<\infty$. Then there are infinitely many graphs $G$ whose order $n$ is divisible by $|H|$ and such that

$$
d(x)+d(y) \geq 2\left(1-\frac{1}{r-\frac{2}{m+2}}\right) n-1
$$

for all non-adjacent $x \neq y \in V(G)$ containing a vertex that does not belong to a copy of $H$. (In particular, $G$ has no perfect $H$-packing.)

Proof. Let $t \in \mathbb{N}$ be such that $((m+2) r-2)(r-2)$ divides $t$. Define $s:=2|H| /((m+$ $2) r-2)$. Let $G^{\prime}$ be the complete $(r+m-1)$-partite graph with one vertex class $V_{1}$ of size 
st $-1, m$ vertex classes $V_{2}, \ldots, V_{m+1}$ of size $s t$ and $r-2$ vertex classes $V_{m+2}, \ldots, V_{r+m-1}$ of size $\frac{|H| t-(m+1) s t}{r-2}$. Let $G$ be obtained from $G^{\prime}$ by adding a vertex $w$ to $G^{\prime}$ such that $w$ is adjacent to precisely those vertices in $V_{m+2} \cup \cdots \cup V_{r+m-1}$. So $|G|=|H| t$.

Any $y \in V_{1} \cup \cdots \cup V_{m+1}$ satisfies

$$
d(y)+d(w) \geq 2|H| t-(m+2) s t-1=2\left(1-\frac{m+2}{(m+2) r-2}\right)|G|-1 .
$$

Furthermore, given any $y_{1} \neq y_{2} \in V_{i}$ for some $m+2 \leq i \leq r+m-1$, we have

$$
\begin{aligned}
d\left(y_{1}\right)+d\left(y_{2}\right) & =2|H| t-2\left(\frac{|H| t-(m+1) s t}{r-2}\right)=2|G|-\frac{2}{r-2}\left(1-\frac{2(m+1)}{(m+2) r-2}\right)|G| \\
& =2|G|-\frac{2}{r-2} \frac{(m+2)(r-2)}{(m+2) r-2}|G|=2\left(1-\frac{m+2}{(m+2) r-2}\right)|G| .
\end{aligned}
$$

Since $d(y)+d\left(y^{\prime}\right) \geq d(y)+d(w)$ for any $y \neq y^{\prime} \in V_{i}$ with $1 \leq i \leq m+1$ this implies that $G$ satisfies our Ore-type degree condition.

Suppose that $w$ belongs to some copy $H_{w}$ of $H$ in $G$. Since $\chi(G)=m+r-1$, an optimal colouring of $G$ induces an $(m+r-1)$-colouring of $H_{w}$ and an $(r-2)$-colouring of $G[N(w)]$. But then $w$ must be playing the role of a vertex $x \in V(H)$ such that $\chi(H[N(x)]) \leq r-2$, contradicting the definition of $m=C E(H)$.

We will now use Propositions 9 and 10 to prove the lower bound of Theorem 2 ,

Proof of Theorem 2 (lower bound). In the case when $\operatorname{hcf}(H) \neq 1$ the lower bound follows from the lower bound in Theorem 1. Proposition 9 settles the case when $C E(H)=\infty$. So we may assume that $\operatorname{hcf}(H)=1$ and $C E(H)<\infty$. In this case, the lower bound in Theorem 1 also implies that

$$
\delta_{\text {Ore }}(H, n) \geq 2\left(1-1 / \chi_{c r}(H)\right) n-2
$$

(for any graph $H$ ). Suppose first that $H$ is bipartite. Since $C E(H)<\infty$ this means that $H$ must have an isolated vertex and so $C E(H)=0$. Thus $\chi_{\text {Ore }}(H)=\chi_{c r}(H)$ and so we are done by (1).

So suppose next that $\chi(H) \geq 3$. In this case the proof of Proposition 10 implies the lower bound whenever $n$ is divisible by $((m+2) r-2)(r-2)|H|$. To deduce the lower bound for any $n \geq((m+2) r-2)(r-2)|H|$ which is divisible by $|H|$ we proceed as follows. Let $n^{\prime}$ be the largest integer such that $n^{\prime} \leq n$ and $n^{\prime}$ is divisible by $((m+2) r-$ $2)(r-2)|H|$. Construct a graph $G$ of order $n^{\prime}$ as in the proof of Proposition 10, Then add $n-n^{\prime}<((m+2) r-2)(r-2)|H|$ new vertices to $V_{1}$ so that these vertices have the same neighbourhoods as the original vertices in $V_{1}$. Then $|G|=n$ and by the same argument as in Proposition 10, $G$ does not contain a perfect $H$-packing. Moreover, it is easy to check that $d(x)+d(y) \geq 2(1-1 /(r-2 /(m+2))) n-2|H|^{4}$ for all non-adjacent $x \neq y \in V(G)$. 


\section{Some USEFul Results}

In Section 2 we proved the lower bound on $\delta_{\text {Ore }}(H, n)$ in Theorem 2 , The following two results together imply the upper bound.

Lemma 11. Let $H$ be a graph and let $\eta>0$. There exists an integer $n_{0}=n_{0}(H, \eta)$ such that if $G$ is a graph whose order $n \geq n_{0}$ is divisible by $|H|$ and

$$
d(x)+d(y) \geq 2\left(1-\frac{1}{\chi(H)}+\eta\right) n
$$

for all non-adjacent $x \neq y \in V(G)$ then $G$ contains a perfect $H$-packing.

Lemma 12. Let $\eta>0$ and suppose that $H$ is a graph such that $\operatorname{hcf}(H)=1$ and $C E(H)<\infty$. There exists an integer $n_{0}=n_{0}(H, \eta)$ such that if $G$ is a graph whose order $n \geq n_{0}$ is divisible by $|H|$ and

$$
d(x)+d(y) \geq \max \left\{2\left(1-\frac{1}{\chi(H)-\frac{2}{C E(H)+2}}+\eta\right) n, 2\left(1-\frac{1}{\chi_{c r}(H)}+\eta\right) n\right\}
$$

for all non-adjacent $x \neq y \in V(G)$ then $G$ contains a perfect $H$-packing.

Note that Lemma 11 implies the upper bound on $\delta(H, n)$ by Alon and Yuster (which we mentioned in Section 1). We now deduce Lemma 11 from Lemma 12

Proof of Lemma 11. Let $h:=|H|$ and $r:=\chi(H)$. Given any $k \geq 2$, define $H^{*}$ to be the complete $(r+1)$-partite graph with one vertex class of size 1 , one vertex class of size $h k-1$ and $r-1$ vertex classes of size $h k$. Let $H^{\prime}$ be obtained from $H^{*}$ by removing an edge between some vertex $y$ in a vertex class of size $h k$ and the vertex in the singleton vertex class. So $\chi\left(H^{\prime}\right)=r+1,\left|H^{\prime}\right|=h k r$ and $\chi\left(H^{\prime}[N(y)]\right)=r-1$. Moreover, $C E\left(H^{\prime}\right)=0$ since $N(y)$ lies in $r-1$ vertex classes of $H^{\prime}$. It is easy to see that $H^{\prime}$ contains a perfect $H$-packing and that $\operatorname{hcf}\left(H^{\prime}\right)=1$. So $\chi_{\text {Ore }}\left(H^{\prime}\right)=\chi_{c r}\left(H^{\prime}\right)=$ $\left(\chi\left(H^{\prime}\right)-1\right) \frac{\left|H^{\prime}\right|}{\left|H^{\prime}\right|-\sigma\left(H^{\prime}\right)}=r \frac{\left|H^{\prime}\right|}{\left|H^{\prime}\right|-1}$. In particular, we can choose $k$ sufficiently large to guarantee that $1 / \chi_{c r}\left(H^{\prime}\right) \geq 1 / \chi(H)-\eta / 4$.

Consider any graph $G$ as in Lemma 11. Choose $a \leq k r$ such that $n-a h$ is divisible by $\left|H^{\prime}\right|=h k r$. Apply Proposition 7 to obtain $a$ disjoint copies of $H$ in $G$. Remove these a copies of $H$ from $G$ to obtain a graph $G^{\prime}$ whose order is divisible by $\left|H^{\prime}\right|$ and which satisfies

$$
d_{G^{\prime}}\left(x_{1}\right)+d_{G^{\prime}}\left(x_{2}\right) \geq 2\left(1-\frac{1}{\chi(H)}+\frac{\eta}{2}\right)\left|G^{\prime}\right| \geq 2\left(1-\frac{1}{\chi_{c r}\left(H^{\prime}\right)}+\frac{\eta}{4}\right)\left|G^{\prime}\right|
$$

for all non-adjacent $x_{1} \neq x_{2} \in V\left(G^{\prime}\right)$. Apply Lemma 12 to find a perfect $H^{\prime}$-packing in $G^{\prime}$. In particular, this induces a perfect $H$-packing in $G^{\prime}$. Thus, together with all those copies of $H$ in $G-G^{\prime}$ we have chosen before, we obtain a perfect $H$-packing in $G$. 
Thus to prove Theorem 2 it remains to prove Lemma 12, which we will do in Section 7 . In order to deal with the 'exceptional' vertices in the proof of Lemma 12 we use the following result which implies that every vertex $w$ of a graph $G$ as in Lemma 12 is contained in a copy of $H$. We prove Lemma 13 in Section 6 .

Lemma 13. Let $H$ be a graph such that $m:=C E(H)<\infty$. Let $r:=\chi(H)$ and $\eta>0$. There exists an integer $n_{0}=n_{0}(\eta, H)$ such that whenever $G$ is a graph on $n \geq n_{0}$ vertices with

$$
d(x)+d(y) \geq 2\left(1-\frac{1}{r-\frac{2}{m+2}}+\eta\right) n
$$

for all non-adjacent $x \neq y \in V(G)$ then every vertex of $G$ lies in a copy of $H$ in $G$.

The above results also imply Theorem 5 :

Proof of Theorem 5. The lower bound in the case when $C E(H)=\infty$ follows from Proposition 9. If $C E(H)<\infty$ and $\chi(H) \geq 3$ then Proposition 10 gives the lower bound for infinitely many values of $n$ and as in the proof of the lower bound in Theorem 2 it can be used to derive the lower bound for any $n$. If $C E(H)<\infty$ and $\chi(H)=2$ then $C E(H)=0$ and so the lower bound is trivial. The upper bound follows from Lemmas 11 and 13 .

In our proof of Lemma 12 we will also use the following result, Lemma 12 from [14. It gives a sufficient condition on the sizes of the vertex classes of a complete $\chi(H)$-partite graph $G$ which ensures that $G$ has a perfect $H$-packing. Lemma 14 is the point where the assumption that $\operatorname{hcf}(H)=1$ is crucial - it is false for graphs with $\operatorname{hcf}(H) \neq 1$.

Lemma 14. Let $H$ be a graph with $\operatorname{hcf}(H)=1$. Put $r:=\chi(H)$ and $\gamma:=(r-$ 1) $\sigma(H) /(|H|-\sigma(H))$. Let $0<\beta_{1} \ll \lambda_{1} \ll \gamma, 1-\gamma, 1 /|H|$ be positive constants. Suppose that $G$ is a complete $r$-partite graph with vertex classes $U_{1}, \ldots, U_{r}$ such that $|G| \gg|H|$ is divisible by $|H|,\left(1-\lambda_{1}^{1 / 10}\right)\left|U_{r}\right| \leq \gamma\left|U_{i}\right| \leq\left(1-\lambda_{1}\right)\left|U_{r}\right|$ for all $i<r$ and such that ||$U_{i}|-| U_{j}|| \leq \beta_{1}|G|$ whenever $1 \leq i<j<r$. Then $G$ contains a perfect $H$-packing.

Here (and later on) we write $0<a_{1} \ll a_{2} \ll a_{3} \leq 1$ to mean that we can choose the constants $a_{1}, a_{2}, a_{3}$ from right to left. More precisely, there are increasing functions $f$ and $g$ such that, given $a_{3}$, whenever we choose some $a_{2} \leq f\left(a_{3}\right)$ and $a_{1} \leq g\left(a_{2}\right)$, all calculations needed in the proof of Lemma 14 are valid.

\section{The Regularity lemma and the Blow-up lemma}

In the proof of Lemma 12 we will use Szemerédi's Regularity lemma 18] and the Blowup lemma of Komlós, Sárközy and Szemerédi [10]. In this section we will introduce all the information we require about these two results. To do this, we firstly introduce some more notation. The density of a bipartite graph $G$ with vertex classes $A$ and $B$ is defined to be

$$
d(A, B):=\frac{e(A, B)}{|A||B|} .
$$

Given any $\varepsilon>0$, we say that $G$ is $\varepsilon$-regular if for all sets $X \subseteq A$ and $Y \subseteq B$ with $|X| \geq \varepsilon|A|$ and $|Y| \geq \varepsilon|B|$ we have $|d(A, B)-d(X, Y)|<\varepsilon$. In this case we also say 
that $(A, B)$ is an $\varepsilon$-regular pair. Given $d \in[0,1)$ we say that $G$ is $(\varepsilon, d)$-super-regular if all sets $X \subseteq A$ and $Y \subseteq B$ with $|X| \geq \varepsilon|A|$ and $|Y| \geq \varepsilon|B|$ satisfy $d(X, Y)>d$ and, furthermore, if $d_{G}(a)>d|B|$ for all $a \in A$ and $d_{G}(b)>d|A|$ for all $b \in B$.

We will use the following degree form of Szemerédi's Regularity lemma which can be easily derived from the classical version.

Lemma 15 (Regularity lemma). For every $\varepsilon>0$ and each integer $\ell_{0}$ there is an $M=$ $M\left(\varepsilon, \ell_{0}\right)$ such that if $G$ is any graph on at least $M$ vertices and $d \in[0,1)$, then there exists a partition of $V(G)$ into $\ell+1$ classes $V_{0}, V_{1}, \ldots, V_{\ell}$, and a spanning subgraph $G^{\prime} \subseteq G$ with the following properties:

- $\ell_{0} \leq \ell \leq M,\left|V_{0}\right| \leq \varepsilon|G|,\left|V_{1}\right|=\cdots=\left|V_{\ell}\right|=: L$,

- $d_{G^{\prime}}(v)>d_{G}(v)-(d+\varepsilon)|G|$ for all $v \in V(G)$,

- $e\left(G^{\prime}\left[V_{i}\right]\right)=0$ for all $i \geq 1$,

- for all $1 \leq i<j \leq \ell$ the graph $\left(V_{i}, V_{j}\right)_{G^{\prime}}$ is $\varepsilon$-regular and has density either 0 or greater than $d$.

The sets $V_{1}, \ldots, V_{\ell}$ are called clusters, $V_{0}$ is called the exceptional set and the vertices in $V_{0}$ exceptional vertices. We refer to $G^{\prime}$ as the pure graph of $G$. Clearly, we may assume that $\left(V_{i}, V_{j}\right)_{G}$ is not $\varepsilon$-regular or has density at most $d$ whenever $\left(V_{i}, V_{j}\right)_{G^{\prime}}$ contains no edges (for all $1 \leq i<j \leq \ell$ ). The reduced graph $R$ of $G$ is the graph whose vertices are $V_{1}, \ldots, V_{\ell}$ and in which $V_{i}$ is adjacent to $V_{j}$ whenever $\left(V_{i}, V_{j}\right)_{G^{\prime}}$ is $\varepsilon$-regular and has density greater than $d$.

A well-known fact is that the minimum degree of a graph $G$ is almost inherited by its reduced graph. We now prove an analogue of this for an Ore-type degree condition.

Lemma 16. Given a constant $c$, let $G$ be a graph such that $d_{G}(x)+d_{G}(y) \geq c|G|$ for all non-adjacent $x \neq y \in V(G)$. Suppose we have applied Lemma 15 with parameters $\varepsilon$ and $d$ to $G$. Let $R$ be the corresponding reduced graph. Then $d_{R}\left(V_{i}\right)+d_{R}\left(V_{j}\right)>(c-2 d-4 \varepsilon)|R|$ for all non-adjacent $V_{i} \neq V_{j} \in V(R)$.

Proof. Let $V_{1}, \ldots, V_{\ell}$ denote the clusters obtained from Lemma 15, Let $L:=\left|V_{1}\right|=$ $\cdots=\left|V_{\ell}\right|$, let $V_{0}$ denote the exceptional set and let $G^{\prime}$ be the pure graph. Set $G^{\prime \prime}:=$ $G^{\prime}-V_{0}$. Consider any pair $V_{i} V_{j}$ of clusters which does not form an edge in $R$. Pick $x \in V_{i}$ and $y \in V_{j}$ such that $x y \notin E(G)$. So $d_{G}(x)+d_{G}(y) \geq c|G|$ and thus $d_{G^{\prime \prime}}(x)+d_{G^{\prime \prime}}(y)>$ $(c-2 d-4 \varepsilon)|G|$. However, by definition of $G^{\prime \prime}$, each cluster containing a neighbour of $x$ in $G^{\prime \prime}$ must be a neighbour of $V_{i}$ in $R$ and the analogue holds for the clusters containing the neighbours of $y$. Thus $d_{R}\left(V_{i}\right)+d_{R}\left(V_{j}\right) \geq\left(d_{G^{\prime \prime}}(x)+d_{G^{\prime \prime}}(y)\right) / L \geq(c-2 d-4 \varepsilon)|R|$, as required.

We will also use the following Embedding lemma. The proof is based on a simple greedy argument, see e.g. Lemma 7.5.2 in [2] or Theorem 2.1 in [12] for details.

Lemma 17 (Embedding lemma). Let $H$ be an $r$-partite graph with vertex classes $X_{1}, \ldots, X_{r}$ and let $\varepsilon, d, n_{0}$ be constants such that $0<1 / n_{0} \ll \varepsilon \ll d, 1 /|H|$. Let $G$ be an $r$-partite graph with vertex classes $V_{1}, \ldots, V_{r}$ of size at least $n_{0}$ such that $\left(V_{i}, V_{j}\right)_{G}$ is $\varepsilon$-regular and has density at least $d$ whenever $H$ contains an edge between $X_{i}$ and $X_{j}$ (for all $1 \leq i<j \leq r$ ). Then $G$ contains a copy of $H$ such that $X_{i} \subseteq V_{i}$. 
The Blow-up lemma of Komlós, Sárközy and Szemerédi [10] states that one can even find a spanning subgraph $H$ in $G$ provided that $H$ has bounded maximum degree and the bipartite pairs forming $G$ are super-regular.

Lemma 18 (Blow-up lemma). Given a graph $R$ with $V(R)=\{1, \ldots, r\}$ and $d, \Delta>0$, there is a constant $\varepsilon_{0}=\varepsilon_{0}(d, \Delta, r)>0$ such that the following holds. Given $L_{1}, \ldots, L_{r} \in$ $\mathbb{N}$ and $0<\varepsilon \leq \varepsilon_{0}$, let $R^{*}$ be the graph obtained from $R$ by replacing each vertex $i \in V(R)$ with a set $V_{i}$ of $L_{i}$ new vertices and joining all vertices in $V_{i}$ to all vertices in $V_{j}$ precisely when $i j \in E(R)$. Let $G$ be a spanning subgraph of $R^{*}$ such that for every $i j \in E(R)$ the bipartite graph $\left(V_{i}, V_{j}\right)_{G}$ is $(\varepsilon, d)$-super-regular. Then $G$ contains a copy of every subgraph $H$ of $R^{*}$ with $\Delta(H) \leq \Delta$.

\section{Sketch proof of Theorem 4}

Let $G$ be a sufficiently large graph on $n$ vertices so that

$$
d(x)+d(y) \geq 2\left(1-\frac{1}{\chi_{c r}(H)}\right) n
$$

for all non-adjacent $x \neq y \in V(G)$. Let $r:=\chi(H)$. Denote by $B$ the complete $r$ partite graph with one vertex class of size $(r-1) \sigma(H)$ and $r-1$ vertex classes of size $|H|-\sigma(H)$. Note that $\chi_{c r}(B)=\chi_{c r}(H)$ and $B$ has a perfect $H$-packing. Thus by considering $B$ instead of $H$ if necessary, it is sufficient to prove the theorem under the added assumption that $H$ is a complete $r$-partite graph with one vertex class of size $\sigma \in \mathbb{N}$ and $r-1$ vertex classes of size $\omega \in \mathbb{N}$. It suffices to consider the case when $\sigma<\omega$. (It is easy to deduce the case $\sigma=\omega$ from this using the same trick as in the proof of Lemma 11.) Let $H^{\prime}$ denote the complete $r$-partite graph with one vertex class of size $\sigma$ and $r-1$ vertex classes of size $\omega-1$.

The proof of Theorem 4 involves repeated applications of the following claim to reduced graphs of $G$.

Claim 19. Let $0<\tau, 1 / \ell_{0} \ll d^{\prime} \ll \gamma, 1 /|H|$. Let $R^{\prime}$ be a graph on $\ell^{\prime} \geq \ell_{0}$ vertices such that $d(x)+d(y) \geq 2\left(1-1 / \chi_{c r}(H)-d^{\prime}\right) \ell^{\prime}$ for all non-adjacent $x \neq y \in V\left(R^{\prime}\right)$. Suppose that the maximum number of vertices in $R^{\prime}$ covered by an $H$-packing is $N \leq(1-\gamma) \ell^{\prime}$. Then $R^{\prime}$ contains a collection of vertex-disjoint copies of $H, H^{\prime}$ and $K_{r}$ which together cover at least $N+\tau \ell^{\prime}$ vertices.

The proof of Claim 19 is almost identical to that of Lemma 15 from 9 . Full details can be found in [19]. Here we briefly outline the proof. Let $\mathcal{L}$ denote the set of vertices not covered by the largest $H$-packing in $R^{\prime}$. Since the subgraph of $R^{\prime}$ induced by $\mathcal{L}$ must contain a small number of edges (else it will contain a copy of $H$ ), most vertices in $\mathcal{L}$ have 'small' degree in this subgraph. Furthermore, all but at most $|H|-1$ of these vertices $x$ have degree at least $\left(1-1 / \chi_{c r}(H)-d^{\prime}\right) \ell^{\prime}$ in $R^{\prime}$ (otherwise we have a copy of $K_{|H|}$ and thus of $H$ in $\mathcal{L}$ ). Now we proceed exactly as in Lemma 15 from [9]. Indeed, for many such vertices $x$ we can combine $x$ with a suitable copy of $H$ in the packing and replace it with a copy of $H^{\prime}$ and a copy of $K_{r}$ containing $x$. Thus we obtain our desired collection of vertex-disjoint copies of $H, H^{\prime}$ and $K_{r}$.

Consider a graph $F$ and $t \in \mathbb{N}$. Let $F(t)$ denote the graph obtained from $F$ by replacing every vertex $x \in V(F)$ by a set $U_{x}$ of $t$ independent vertices, and joining each 
$u \in U_{x}$ to each $v \in U_{y}$ precisely when $x y$ is an edge in $F$. In other words we replace the edges of $F$ by copies of $K_{t, t}$. We will refer to $F(t)$ as a blown-up copy of $F$.

Define constants

$$
0 \ll 1 / \ell_{0} \ll \varepsilon \ll \varepsilon^{\prime} \ll d \ll d^{\prime} \ll \gamma \ll \eta .
$$

Apply the Regularity lemma with parameters $\varepsilon, d$ and $\ell_{0}$ to $G$ to obtain clusters $V_{1}, \ldots, V_{\ell}$ of size $L$, an exceptional set $V_{0}$ and a reduced graph $R$. By Lemma 16 we have that

$$
d_{R}\left(V_{i}\right)+d_{R}\left(V_{j}\right) \geq 2\left(1-\frac{1}{\chi_{c r}(H)}-2 d\right)|R|
$$

for all $V_{i} \neq V_{j}$ with $V_{i} V_{j} \notin E(R)$.

We wish to find an $H$-packing either in $R$ or in a blown-up copy of $R$, which covers at least a $(1-\gamma)$-proportion of the clusters. Let $N$ denote the maximum number of clusters in $R$ covered by an $H$-packing. If $N \geq(1-\gamma) \ell$ we are done. If not, then by Claim 19 $R$ contains a collection of vertex-disjoint copies of $H, H^{\prime}$ and $K_{r}$ which together cover at least $N+\tau \ell$ vertices. Let

$$
t:=(\omega-\sigma)|H| .
$$

It is not hard to see that $H(t), H^{\prime}(t)$ and $K_{r}(t)$ all have perfect $H$-packings. Thus $R_{1}:=R(t)$ contains an $H$-packing covering at least $(N+\tau \ell) t$ vertices. Since $\left|R_{1}\right|=\ell t$, a larger proportion of the vertices in $R_{1}$ are covered by this $H$-packing compared to the $H$-packing in $R$. If $(N+\tau \ell) t \geq(1-\gamma) \ell t$ we are done. Otherwise we can continue this process: By applying Claim 19 to $R_{1}$ we see that $R_{1}(t)=R\left(t^{2}\right)$ contains an $H$-packing covering a substantially larger proportion of the vertices than the previous $H$-packing. Eventually we obtain a graph $R^{\prime}$, where $R^{\prime}=R\left(t^{k}\right)$ for some constant $k=k\left(H, \gamma, d^{\prime}\right)$, such that there is an $H$-packing $\mathcal{H}$ covering at least $(1-\gamma)\left|R^{\prime}\right|$ clusters in $R^{\prime}$.

Suppose that $(A, B)$ is an $\varepsilon$-regular pair of density at least $d$. By removing at most $t^{k}$ vertices from $A$ and $B$, we can partition both $A$ and $B$ into $t^{k}$ equal subclusters $A_{1}, \ldots, A_{t^{k}}$ and $B_{1}, \ldots, B_{t^{k}}$ respectively. We can do this in such a way that each $\left(A_{i}, B_{j}\right)$ is an $\varepsilon^{\prime}$-regular pair with density at least $d-\varepsilon$. So since each edge of $R$ corresponds to an $\varepsilon$-regular pair of density at least $d$, the edges of $R^{\prime}$ can be viewed as corresponding to $\varepsilon^{\prime}$-regular pairs with density at least $d-\varepsilon$. (Thus for each $V_{i} \in V(R)$ there are $t^{k}$ vertices in $R^{\prime}$ which correspond to subclusters of $V_{i}$.)

Suppose that $H^{*}$ is a copy of $H$ in $\mathcal{H}$. Consider the subgraph $H_{G}^{*}$ of $G$ whose vertex set consists of all those vertices lying in the clusters of $H^{*}$, and whose edge set consists of all those edges which lie in an $\varepsilon^{\prime}$-regular pair corresponding to an edge in $H^{*}$. It is easy to see (for example by repeated use of Lemma 17) that there is an $H$-packing covering almost all the vertices in $H_{G}^{*}$. We find such an $H$-packing for each copy of $H$ in $\mathcal{H}$. Since $\mathcal{H}$ covers at least $(1-\gamma)\left|R^{\prime}\right|$ of the clusters in $R^{\prime}$, and since $\gamma \ll \eta$, the union of all these $H$-packings covers at least $(1-\eta) n$ vertices of $G$, as desired.

\section{Proof of Lemma 13}

Let $H$ be as in the statement of the lemma and let $G$ be a graph of sufficiently large order $n$ which satisfies (3). Recall that $r=\chi(H)$ and $m=C E(H)$. Let $x$ be any vertex of $G$. We have to find a copy of $H$ in $G$ which contains $x$. Suppose first that $r=2$. Then $H$ must have an isolated vertex $v$ (since $C E(H)<\infty)$. So we can apply 
Proposition 7 to find a copy of $H-v$ in $G-x$ and thus a copy of $H$ in $G$ (where $x$ plays the role of $v$ ).

So suppose that $r \geq 3$. Choose additional constants $\varepsilon, d$ and $\alpha$ such that

$$
0<\varepsilon \ll d \ll \alpha \ll \eta
$$

and let $\ell_{0}:=1 / \varepsilon$. Apply the Regularity lemma with parameters $\varepsilon, d, \ell_{0}$ to $G$ to obtain clusters $V_{1}, \ldots, V_{\ell}$ of size $L$, an exceptional set $V_{0}$, a pure graph $G^{\prime}$ and a reduced graph $R$. Let

$$
k:=(m+2) r-2 .
$$

Lemma 16 implies that

$$
d_{R}\left(V_{i}\right)+d_{R}\left(V_{j}\right) \geq 2\left(1-\frac{1}{r-\frac{2}{m+2}}+\frac{\eta}{2}\right)|R|=2\left(1-\frac{m+2}{k}+\frac{\eta}{2}\right)|R|
$$

for all $V_{i} \neq V_{j} \in V(R)$ with $V_{i} V_{j} \notin E(R)$. By adding the vertices of one cluster to $V_{0}$ if necessary (and deleting this cluster from $R$ ) we may assume that $x \in V_{0}$. (So now $\left|V_{0}\right| \leq 2 \varepsilon n$.) We say that $x$ is adjacent to a cluster $V_{i} \in V(R)$ if $x$ is adjacent to at least $\alpha L$ vertices of $V_{i}$ in $G$. We denote by $S$ the set of clusters $V_{i} \in V(R)$ that $x$ is adjacent to, and define $s:=|S| /|R|$. Also, we write $\bar{S}:=V(R) \backslash S$. Note that

$$
d_{G}(x) \leq|S| L+|\bar{S}| \alpha L+\left|V_{0}\right| \leq(s+\alpha+2 \varepsilon) n \leq(s+2 \alpha) n
$$

and so

$$
s \geq \frac{\delta(G)}{n}-2 \alpha \stackrel{(3)}{\geq}\left(1-\frac{2}{r-\frac{2}{m+2}}+2 \eta\right)-2 \alpha \geq 1-\frac{2(m+2)}{k}+\eta .
$$

In particular $s>0$ since $r \geq 3$. Our aim now is to find either a copy $K_{r}^{\prime}$ of $K_{r}$ in $R$ containing $r-1$ clusters adjacent to $x$ (i.e. $\left|V\left(K_{r}^{\prime}\right) \cap S\right| \geq r-1$ ), or a copy $K_{r+m}^{\prime}$ of $K_{r+m}$ in $R$ containing $r-2$ clusters adjacent to $x$. In both cases we could apply the Embedding lemma (Lemma 17) to find the desired copy $H_{x}$ of $H$ in $G$. Indeed, in the case where we find $K_{r+m}^{\prime}$ we could use $x$ to play the role of a vertex $y \in V(H)$ for which there exists an $(r-2)$-colouring of $H[N(y)]$ that can be extended to an $(r+m)$-colouring of $H$. The neighbourhood $N_{H}(y)$ of $y$ would be embedded into the clusters belonging to $V\left(K_{r+m}^{\prime}\right) \cap S$ and $H-N_{H}(y)$ would be embedded into the clusters belonging to $V\left(K_{r+m}^{\prime}\right)$ (so here we use the fact that $C E(H)=m$ ). In the case where we find $K_{r}^{\prime}, x$ can play the role of any vertex of $H$. Given some optimal colouring of $H$, the vertices of $H$ which have a different colour than $x$ are embedded into the clusters in $V\left(K_{r}^{\prime}\right) \cap S$ (so we only use that $\chi(H)=r$ in this case).

Let $C$ be the set of clusters $U \in S$ with $d_{R}(U)<(1-(m+2) / k+\eta / 2)|R|$. By (4), $C$ induces a clique. So we may assume that $|C|<r$, since otherwise we have our copy $K_{r}^{\prime}$ of $K_{r}$. Suppose now that for some $1 \leq i \leq r-1$ we have already found $i$ clusters $U_{1}, \ldots, U_{i} \in S \backslash C$ such that $U_{1}, \ldots, U_{i}$ form a copy $K_{i}^{\prime}$ of $K_{i}$ in $R$. Then

$$
\left|\bigcap_{1 \leq j \leq i} N_{R}\left(U_{j}\right)\right| \geq-(i-1)|R|+\sum_{j=1}^{i} d_{R}\left(U_{j}\right) \geq\left(1-\frac{i(m+2)}{k}+\eta / 2\right)|R| .
$$

Case 1. $1-s \leq(2 m+2) / k$ 
In this case, we will find a copy of $K_{r}$ which contains at least $r-1$ vertices in $S$. Suppose that $i \leq r-2$ and we have found $U_{1}, \ldots, U_{i}$ as above. Then $1-i(m+2) / k \geq(2 m+2) / k$ and so (7) implies that the common neighbourhood $N_{R}\left(K_{i}^{\prime}\right)$ of $K_{i}^{\prime}$ satisfies $\left|N_{R}\left(K_{i}^{\prime}\right)\right| \geq$ $(1-s+\eta / 2)|R|$. So we can choose $U_{i+1} \in S \backslash C$ to extend $K_{i}^{\prime}$ into a copy of $K_{i+1}$ in $R[S \backslash C]$ (we can avoid $C$ when choosing $U_{i+1}$ since $|C|<r \ll \eta|R|$ ). If $i=r-1$, then $1-\frac{i(m+2)}{k}=\frac{m}{k} \geq 0$. So $\left|N_{R}\left(K_{i}^{\prime}\right)\right| \geq \eta|R| / 2$ and we can extend $K_{i}^{\prime}=K_{r-1}^{\prime}$ into the desired copy of $K_{r}$ using an arbitrary vertex of $R$.

Case 2. $1-s \geq(2 m+2) / k$

In this case, we will either find a copy of $K_{r}$ which contains at least $r-1$ vertices in $S$ or find a copy of $K_{r+m}$ which contains at least $r-2$ vertices in $S$. Suppose that $i \leq r-3$ and we have found $U_{1}, \ldots, U_{i}$ as described before Case 1 which form a copy $K_{i}^{\prime}$ of $K_{i}$ in $R[S \backslash C]$. Note that

$$
1-\frac{i(m+2)}{k} \geq \frac{k-(r-3)(m+2)}{k}=\frac{3(m+2)-2}{k} \geq \frac{2(m+2)}{k} \stackrel{(6)}{\geq} 1-s .
$$

Thus (77) implies that we can choose a cluster $U_{i+1} \in S \backslash C$ which forms a $K_{i+1}$ together with $K_{i}^{\prime}$. This shows that we can find a copy $K_{r-2}^{\prime}$ of $K_{r-2}$ which lies in $R[S \backslash C]$. Note that (7) also implies that the common neighbourhood $N_{R}\left(K_{r-2}^{\prime}\right)$ of $K_{r-2}^{\prime}$ satisfies

$$
\left|N_{R}\left(K_{r-2}^{\prime}\right)\right| \geq\left(1-\frac{(r-2)(m+2)}{k}+\frac{\eta}{2}\right)|R|=\left(\frac{2(m+1)}{k}+\frac{\eta}{2}\right)|R| .
$$

Now we aim to extend $K_{r-2}^{\prime}$ into a copy $K_{r+m}^{\prime}$ of $K_{r+m}$. We will aim to find the additional vertices in $\bar{S}$. Suppose for some $0 \leq i \leq m+1$ we have found $i$ clusters $W_{1}, \ldots, W_{i} \in \bar{S}$ which together with $K_{r-2}^{\prime}$ form a copy $K_{r-2+i}^{\prime}$ of $K_{r-2+i}$ in $R$. We will need a lower bound on $d_{R}\left(W_{j}\right)$ for all $j=1, \ldots, i$. To derive this, note that the definition of $S$ implies that $W_{j}$ contains a vertex $y$ which is not adjacent to $x$ in $G$. So (3) and (5) and the inequality in Case 2 imply that

$$
d_{G}(y) \geq\left(2\left(1-\frac{m+2}{k}+\eta\right)-s-2 \alpha\right) n \geq\left(1-\frac{2}{k}+\eta\right) n
$$

and so $d_{G^{\prime}}(y) \geq(1-2 / k+\eta / 2) n$. But each cluster containing a neighbour of $y$ in $G^{\prime}$ must be a neighbour of $W_{j}$ in $R$. Hence

$$
d_{R}\left(W_{j}\right) \geq \frac{d_{G^{\prime}}(y)-\left|V_{0}\right|}{L} \geq\left(1-\frac{2}{k}\right)|R| .
$$

So the common neighbourhood $N_{R}\left(K_{r-2+i}^{\prime}\right)$ of $K_{r-2+i}^{\prime}$ satisfies

$$
\left|N_{R}\left(K_{r-2+i}^{\prime}\right)\right| \geq\left|N_{R}\left(K_{r-2}^{\prime}\right)\right|-i|R|+\sum_{j=1}^{i} d_{R}\left(W_{j}\right) \stackrel{(8),(9)}{\geq}\left(\frac{2(m+1)}{k}-i \frac{2}{k}+\frac{\eta}{2}\right)|R| \geq \frac{\eta|R|}{2} .
$$

So we can choose a vertex $W_{i+1} \in V(R) \backslash C$ that is a common neighbour of the clusters in $K_{r-2+i}^{\prime}$. Suppose that $W_{i+1} \in S$. Then together with $K_{r-2}^{\prime}$ this forms a copy $K_{r-1}^{\prime}$ of $K_{r-1}$ in $R[S \backslash C]$. Now (7) implies that $\left|N_{R}\left(K_{r-1}^{\prime}\right)\right| \geq(m / k+\eta / 2)|R|$ and so we can extend $K_{r-1}^{\prime}$ to a copy of $K_{r}$ with at least $r-1$ vertices in $S$. So we may assume that 
$W_{i+1} \in \bar{S}$. Continuing in this way, we obtain a copy of $K_{r+m}$ having $r-2$ clusters in $S$, as required.

\section{Proof of Lemma 12}

7.1. Preliminaries and an outline of the proof. Let $H, G$ and $\eta>0$ be as in Lemma 12 and let $r:=\chi(H)$. Choose $t \in \mathbb{N}$ such that $t|H|(r-1) \geq 4 r / \eta$. Let $z_{1}:=t(r-1) \sigma(H)$ and $z:=t(|H|-\sigma(H))$. Put $\gamma:=z_{1} / z$. Note that $0<\gamma<1$ since $\operatorname{hcf}(H)=1$. Define $B^{*}$ to be the complete $r$-partite graph with one vertex class of size $z_{1}$ and $r-1$ vertex classes of size $z$. Then $B^{*}$ has a perfect $H$-packing and $\eta\left|B^{*}\right| / 4 \geq r$. Moreover,

$$
\chi_{c r}\left(B^{*}\right)=\chi_{c r}(H)=(r-1) \frac{|H|}{|H|-\sigma(H)}=r-1+\frac{(r-1) \sigma(H)}{|H|-\sigma(H)}=r-1+\gamma .
$$

Choose $s \in \mathbb{N}$ and a new constant $\lambda$ such that $0<\lambda \ll \eta, \gamma, 1-\gamma$ as well as $s_{1}:=$ $\gamma(1+\lambda) s \in \mathbb{N}$ and $s_{1} \leq s$. Let $B^{\prime}$ denote the complete $r$-partite graph with one vertex class of size $s_{1}$ and $r-1$ vertex classes of size $s$. Thus,

$$
\chi_{c r}\left(B^{\prime}\right)=(r-1) \frac{\left|B^{\prime}\right|}{\left|B^{\prime}\right|-s_{1}}=r-1+\gamma(1+\lambda) .
$$

Note that the proportion $\gamma(1+\lambda)$ of the size of the smallest vertex class of $B^{\prime}$ compared to the size of one of the larger classes is slightly larger than the corresponding proportion $\gamma$ associated with $B^{*}$. We can therefore choose $s$ and $\lambda$ in such a way that $B^{\prime}$ has a perfect $B^{*}$-packing, and thus a perfect $H$-packing. (Indeed, the perfect $B^{*}$-packing would consist of 'most' but not all of the copies of $B^{*}$ having their smallest vertex class lying in the smallest vertex class of $B^{\prime}$.)

We now give an outline for the proof of Lemma 12. We first apply the Regularity lemma to $G$ to obtain a reduced graph $R$. Since $R$ almost inherits the Ore-type condition on $G$ we may apply Theorem 4 to find an almost perfect $B^{\prime}$-packing of $R$. We then remove all clusters from $R$ that are not covered by this $B^{\prime}$-packing and add the vertices in these clusters to the exceptional set $V_{0}$.

For each exceptional vertex $x \in V_{0}$, we apply Lemma 13 to find a copy of $H$ in $G$ containing $x$, and remove the vertices in this copy from $G$. Thus some vertices in clusters in $R$ will be removed from $G$. The copies of $H$ will be chosen to be disjoint for different exceptional vertices.

Our aim is to apply the Blow-up lemma to each copy $B_{i}^{\prime}$ of $B^{\prime}$ in the $B^{\prime}$-packing of $R$ in order to find an $H$-packing in $G$ which covers all the vertices belonging to (the modified) clusters in $B_{i}^{\prime}$. Then all these $H$-packings together with all those copies of $H$ chosen for the exceptional vertices would form a perfect $H$-packing in $G$. However, to do this, we need that the complete $r$-partite graph $F_{i}^{*}$ whose $j$ th vertex class is the union of all the clusters in the $j$ th vertex class of $B_{i}^{\prime}$ has a perfect $H$-packing. Lemma 14 gives a condition which guarantees this.

To apply Lemma 14 we need that $\left|F_{i}^{*}\right|$ is divisible by $|H|$. We will remove a bounded number of further copies of $H$ from $G$ to ensure this (see Section 7.4). Furthermore, we require that $F_{i}^{*}$ has $r-1$ vertex classes of roughly the same size, $u$ say, and that its other vertex class is a little larger than $\gamma u$. But this condition will be satisfied automatically 
by the choice of the sizes of the vertex classes in $B^{\prime}$. In fact, this is the reason why we chose a $B^{\prime}$-packing in $R$ rather than a $B^{*}$-packing. The above strategy is based on that in [13. However, there are additional difficulties.

7.2. Applying the Regularity lemma and modifying the reduced graph. We define further constants satisfying

$$
0<\varepsilon \ll d \ll \eta_{1} \ll \beta \ll \alpha \ll \lambda \ll \eta, \gamma, 1-\gamma .
$$

We also choose $\eta_{1}$ so that

$$
\eta_{1} \ll \frac{1}{\left|B^{\prime}\right|} .
$$

Throughout the proof we assume that the order $n$ of our graph $G$ is sufficiently large for our calculations to hold. Apply the Regularity lemma with parameters $\varepsilon, d$ and $\ell_{0}:=1 / \varepsilon$ to obtain clusters $V_{1}, \ldots, V_{\ell}$ of size $L$, an exceptional set $V_{0}$, a pure graph $G^{\prime}$ and a reduced graph $R$. Let $m:=C E(H)$. By Lemma 16 we have that

$$
d_{R}\left(V_{j_{1}}\right)+d_{R}\left(V_{j_{2}}\right) \geq \max \left\{2\left(1-\frac{1}{r-\frac{2}{m+2}}+\frac{\eta}{2}\right)|R|, 2\left(1-\frac{1}{\chi_{c r}(H)}+\frac{\eta}{2}\right)|R|\right\}
$$

for all $V_{j_{1}} \neq V_{j_{2}} \in V(R)$ with $V_{j_{1}} V_{j_{2}} \notin E(R)$. Together with (11) and (12) this implies that

$$
d_{R}\left(V_{j_{1}}\right)+d_{R}\left(V_{j_{2}}\right) \geq 2\left(1-\frac{1}{\chi_{c r}\left(B^{\prime}\right)}\right)|R|
$$

for all $V_{j_{1}} \neq V_{j_{2}} \in V(R)$ with $V_{j_{1}} V_{j_{2}} \notin E(R)$. So we can apply Theorem 4 to $R$ to obtain a $B^{\prime}$-packing covering all but at most $\eta_{1}|R|$ vertices. We denote the copies of $B^{\prime}$ in this packing by $B_{1}^{\prime}, \ldots, B_{\ell^{\prime}}^{\prime}$. We delete all the clusters not contained in some $B_{i}^{\prime}$ from $R$ and add all vertices lying in these clusters to $V_{0}$. So $\left|V_{0}\right| \leq \varepsilon n+\eta_{1} n \leq 2 \eta_{1} n$. We now refer to $R$ as this modified reduced graph. We still have that

$$
d_{R}\left(V_{j_{1}}\right)+d_{R}\left(V_{j_{2}}\right) \geq \max \left\{2\left(1-\frac{1}{r-\frac{2}{m+2}}+\frac{\eta}{4}\right)|R|, 2\left(1-\frac{1}{\chi_{c r}(H)}+\frac{\eta}{4}\right)|R|\right\}
$$

for all $V_{j_{1}} \neq V_{j_{2}} \in V(R)$ with $V_{j_{1}} V_{j_{2}} \notin E(R)$. Recall that by definition of $B^{\prime}$, each $B_{i}^{\prime}$ contains a perfect $B^{*}$-packing. Fix such a $B^{*}$-packing for each $i=1, \ldots, \ell^{\prime}$. The union of all these $B^{*}$-packings gives us a perfect $B^{*}$-packing $\mathcal{B}^{*}$ in $R$.

Given any $B_{i}^{\prime}$, it is easy to check that we can replace each cluster $V_{j} \in V\left(B_{i}^{\prime}\right)$ with a subcluster of size $L^{\prime}:=\left(1-\varepsilon\left|B^{\prime}\right|\right) L$ such that for each edge $V_{j_{1}} V_{j_{2}}$ of $B_{i}^{\prime}$ the chosen subclusters of $V_{j_{1}}$ and $V_{j_{2}}$ form a $(2 \varepsilon, d / 2)$-super-regular pair in $G^{\prime}$. We do this for each $i=1, \ldots, \ell^{\prime}$ and add all the vertices not belonging to our chosen subclusters to $V_{0}$. We now refer to these subclusters as the clusters of $R$. Then for every edge $V_{j_{1}} V_{j_{2}}$ of $R$ the pair $\left(V_{j_{1}}, V_{j_{2}}\right)_{G^{\prime}}$ is still $2 \varepsilon$-regular and has density more than $d / 2$. Moreover,

$$
\left|V_{0}\right| \leq 2 \eta_{1} n+\varepsilon\left|B^{\prime}\right| n \leq 3 \eta_{1} n .
$$

We now partition each cluster $V_{j}$ into a red part $V_{j}^{\text {red }}$ and a blue part $V_{j}^{\text {blue }}$ where ||$V_{j}^{\text {red }}|-| V_{j}^{\text {blue }}|| \leq \varepsilon L^{\prime}$ and ||$N_{G}(x) \cap V_{j}^{\text {red }}|-| N_{G}(x) \cap V_{j}^{\text {blue }}|| \leq \varepsilon L^{\prime}$ for all $x \in V(G)$. (Consider a random partition to see that there are $V_{j}^{\text {red }}$ and $V_{j}^{b l u e}$ with these properties.) 
Together all these partitions of the clusters yield a partition of $V(G)-V_{0}$ into a set $V^{r e d}$ of red vertices and a set $V^{\text {blue }}$ of blue vertices. In Section 7.3 we will choose certain copies of $H$ in $G$ to cover the exceptional vertices in $V_{0}$, but each of these copies will avoid the red vertices. All the vertices contained in these copies of $H$ will be removed from the clusters they belong to. However, for every edge $V_{j_{1}} V_{j_{2}}$ of $B_{i}^{\prime}$ the modified bipartite subgraph of $G^{\prime}$ whose vertex classes are the remainders of $V_{j_{1}}$ and $V_{j_{2}}$ will still be $(5 \varepsilon, d / 5)$-super-regular since it still contains all vertices in $V_{j_{1}}^{r e d} \cup V_{j_{2}}^{r e d}$. Furthermore, all edges in $R$ will still correspond to $5 \varepsilon$-regular pairs of density more than $d / 5$. After Section 7.3 we will only remove a bounded number of further vertices from the clusters, which will not affect the super-regularity significantly.

7.3. Incorporating the exceptional vertices. In this section we cover all the exceptional vertices with vertex-disjoint copies of $H$. Let $G^{\text {blue }}$ denote the induced subgraph of $G$ with vertex set $V^{\text {blue }} \cup V_{0}$. The definition of $V^{\text {blue }}$ (2) and (14) together imply that

$d_{G^{\text {blue }}}(x)+d_{G^{\text {blue }}}(y) \geq \max \left\{2\left(1-\frac{1}{r-\frac{2}{m+2}}+\frac{\eta}{2}\right)\left|G^{\text {blue }}\right|, 2\left(1-\frac{1}{\chi_{c r}(H)}+\frac{\eta}{2}\right)\left|G^{\text {blue }}\right|\right\}$

for all non-adjacent $x \neq y \in V\left(G^{b l u e}\right)$. Let $v_{1}, \ldots, v_{\left|V_{0}\right|}$ be an enumeration of the exceptional vertices. Lemma 13 gives us a copy $H_{v_{1}}$ of $H$ in $G^{\text {blue }}$ covering $v_{1}$. Delete the vertices of $H_{v_{1}}$ from $G^{b l u e}$ and apply the lemma again to find a copy $H_{v_{2}}$ of $H$ covering $v_{2}$. We would like to continue this way. However, for later purposes it is convenient to be able to assume that from each cluster we only delete a small proportion of vertices during this process. So before choosing the copy $H_{v_{j}}$ for $v_{j}$ (say), we call $B_{i}^{\prime}$ bad if it contains a cluster meeting the copies $H_{v_{1}}, \ldots, H_{v_{j-1}}$ that we have chosen before in at least $\beta L^{\prime}$ vertices. So at most $\left|V_{0}\right||H| /\left(\beta L^{\prime}\right) \leq 3 \eta_{1}|H| n /\left(\beta L^{\prime}\right) \leq \eta \ell^{\prime} / 10$ of the $B_{i}^{\prime}$ are bad. We delete all the vertices belonging to clusters in bad $B_{i}^{\prime}$ from $G^{b l u e}$. Since there are at most $\eta n / 10 \leq \eta\left|G^{b l u e}\right| / 4$ such vertices, we can still apply Lemma 13 to find $H_{v_{j}}$. Thus we can cover all the exceptional vertices. We remove all the vertices lying in the copies $H_{v_{1}}, \ldots, H_{v_{\left|V_{0}\right|}}$ of $H$ from the clusters they belong to (and from $G$ ).

7.4. Making the blow-up of each $B \in \mathcal{B}^{*}$ divisible by $|H|$. Given a subgraph $S \subseteq R$ we write $V_{G}(S)$ for the set of all those vertices of $G$ that belong to a cluster in $S$. Our aim now is to find, for each $B_{i}^{\prime}$ in our $B^{\prime}$-packing in $R$, an $H$-packing in $G$ covering all the vertices in $V_{G}\left(B_{i}^{\prime}\right)$. Thus, taking the union of these $H$-packings and the copies of $H$ containing the vertices in $V_{0}$, we will obtain a perfect $H$-packing in $G$. If we can ensure that the complete $r$-partite graph whose $j$ th vertex class is the union of all clusters in the $j$ th vertex class of $B_{i}^{\prime}$ has a perfect $H$-packing, then by the Blowup lemma the subgraph of $G^{\prime}$ corresponding to $B_{i}^{\prime}$ will have a perfect $H$-packing. By Lemma 14 the former will turn out to be the case provided that $|H|$ divides $\left|V_{G}\left(B_{i}^{\prime}\right)\right|$. So our next aim is to remove a bounded number of copies of $H$ from $G$ to ensure that $\left|V_{G}\left(B_{i}^{\prime}\right)\right|$ is divisible by $|H|$ for all $i=1, \ldots, \ell^{\prime}$. This in turn will be achieved by ensuring that $|H|$ divides $\left|V_{G}(B)\right|$ for all $B \in \mathcal{B}^{*}$.

Consider the auxiliary graph $F$ whose vertices are the elements of $\mathcal{B}^{*}$ where $B_{1}, B_{2} \in$ $\mathcal{B}^{*}$ are adjacent in $F$ if $R$ contains a copy of $K_{r}$ with one vertex in $B_{1}$ and $r-1$ vertices in $B_{2}$ or vice versa. 
Suppose first that $F$ is connected. Consider a spanning tree $T$ of $F$ with root $B_{0} \in \mathcal{B}^{*}$, say. If $B_{1}, B_{2} \in \mathcal{B}^{*}$ are adjacent in $F$ then by the Embedding lemma $G$ contains a copy of $H$ with one vertex in $V_{G}\left(B_{1}\right)$ and all the other vertices in $V_{G}\left(B_{2}\right)$, or vice versa. (To see this, let $K_{r}^{\prime}$ be a copy of $K_{r}$ in $R$ with one vertex $V \in V_{R}\left(B_{1}\right)$ and all other vertices in $V_{R}\left(B_{2}\right)$. Choose any $V^{\prime} \in V_{R}\left(B_{2}\right)$ which is adjacent to all of $V\left(K_{r}^{\prime}\right) \backslash\{V\}$. Then our copy of $H$ will have one vertex, $v$ say, in $V$. All other vertices of $H$ lying in the same colour class as $v$ will be embedded into $V^{\prime}$ and all the remaining vertices of $H$ will be embedded into $V\left(K_{r}^{\prime}\right) \backslash\{V\}$.) In fact, we can choose $|H|-1$ disjoint such copies of $H$. So by removing at most $|H|-1$ such copies of $H$ we can ensure $\left|V_{G}\left(B_{1}\right)\right|$ is divisible by $|H|$.

We can use this observation to 'shift the remainders mod $|H|$ ' along $T$ to achieve that $|H|$ divides $\left|V_{G}(B)\right|$ for all $B \in \mathcal{B}^{*}$ as follows. Let $j_{\max }$ be the largest distance of some $B \in \mathcal{B}^{*}$ from $B_{0}$ in $T$. Then for all $B \in \mathcal{B}^{*}$ of distance $j_{\text {max }}$ from $B_{0}$ we can remove copies of $H$ as indicated above to ensure that $|H|$ divides $\left|V_{G}(B)\right|$. We can repeat this for all those $B \in \mathcal{B}^{*}$ of distance $j_{\max }-1$ from $B_{0}$ etc. until $\left|V_{G}(B)\right|$ is divisible by $|H|$ for all $B \in \mathcal{B}^{*}$. (This follows as $\sum_{B \in \mathcal{B}^{*}}\left|V_{G}(B)\right|$ is divisible by $|H|$ since $|G|$ is divisible by $|H|$.)

So we may assume that $F$ is not connected. Let $\mathcal{C}$ denote the set of all components of $F$. Given $C \in \mathcal{C}$, we denote by $V_{R}(C) \subseteq V(R)$ the set of all those clusters which belong to some $B \in \mathcal{B}^{*}$ with $B \in C$. We write $V_{G}(C) \subseteq V(G)$ for the union of all the clusters in $V_{R}(C)$. We will show that we can remove a bounded number of copies of $H$ from $G$ to achieve that $\left|V_{G}(C)\right|$ is divisible by $|H|$ for all $C \in \mathcal{C}$. As in the case when $F$ is connected, we can then 'shift the remainders $\bmod |H|$ ' along a spanning tree of each component to make $\left|V_{G}(B)\right|$ divisible by $|H|$ for all $B \in \mathcal{B}^{*}$.

In the case when $r=2$ this is straightforward. Indeed, in this case $H$ contains an isolated vertex (since $C E(H)<\infty$ ). So given any $C \in \mathcal{C}$ we can apply the Embedding lemma to find $|H|-1$ vertex-disjoint copies of $H$ in $G$ such that one vertex (playing the role of the isolated vertex) lies in $V_{G}(C)$ and the other vertices lie in $V_{G}\left(C^{\prime}\right)$ for some $C^{\prime} \in \mathcal{C} \backslash\{C\}$. By removing a suitable number of such copies we can ensure that $|H|$ divides $\left|V_{G}(C)\right|$. Since in the above argument we can choose any $C^{\prime} \in \mathcal{C} \backslash\{C\}$ to contain the remaining vertices of our copy of $H$ (and since $|G|$ is divisible by $|H|$ ) we can apply this argument repeatedly to make $\left|V_{G}\left(C^{\prime \prime}\right)\right|$ divisible by $|H|$ for all $C^{\prime \prime} \in \mathcal{C}$.

So now we consider the case when $r \geq 3$. We need the following claim.

Claim 20. Let $C_{1}, C_{2} \in \mathcal{C}$ and let $V \in V_{R}\left(C_{2}\right)$. Then

$$
\left|N_{R}(V) \cap V_{R}\left(C_{1}\right)\right|<\left(1-\frac{1}{r-1+\gamma}\right)\left|V_{R}\left(C_{1}\right)\right| .
$$

Proof. Suppose not. Then there exists some $B \in \mathcal{B}^{*}$ such that $B \in C_{1}$ and

$$
\left|N_{R}(V) \cap B\right| \geq\left(1-\frac{1}{r-1+\gamma}\right)|B|=|B|-\frac{(r-1) z+z_{1}}{r-1+z_{1} / z}=|B|-z .
$$

Hence $V$ has a neighbour in at least $r-1$ vertex classes of $B$. So $R$ contains a copy of $K_{r}$ with one vertex, namely $V$, in a copy $B_{0} \in \mathcal{B}^{*}$ and $r-1$ vertices in $B$. So $B$ and $B_{0}$ are adjacent in $F$. But they lie in different components of $F$, a contradiction. 
We now show that we can remove a bounded number of copies of $H$ from $G$ to make $\left|V_{G}(C)\right|$ divisible by $|H|$ for some $C \in \mathcal{C}$. (In particular, if $F$ consists of exactly two components $C$ and $C^{\prime}$ this also ensures that $\left|V_{G}\left(C^{\prime}\right)\right|$ is divisible by $|H|$.)

Claim 21. There exists a component $C \in \mathcal{C}$ with $\left|V_{R}(C)\right| \leq|R| / 2$ for which we can ensure that $|H|$ divides $\left|V_{G}(C)\right|$ by removing at most $|H|-1$ copies of $H$ from $G$.

Proof. To prove the claim we will distinguish two cases.

Case 1. There exists a component $C_{1} \in \mathcal{C}$ with $\left|V_{R}\left(C_{1}\right)\right| \leq|R| / 2$ and such that there is a cluster $V_{1} \in V_{R}\left(C_{1}\right)$ with $d_{R}\left(V_{1}\right) \geq\left(1-1 / \chi_{c r}(H)+\eta / 4\right)|R|$.

Recall that $K_{r+1}^{-}$is a $K_{r+1}$ with one edge removed. We call the two non-adjacent vertices of $K_{r+1}^{-}$small. We say that a copy $K^{\prime}$ of $K_{r+1}^{-}$in $R$ is good if either (i) $V\left(K^{\prime}\right) \cap V_{R}\left(C_{1}\right)$ consists of a small vertex of $K^{\prime}$ or (ii) $V\left(K^{\prime}\right) \backslash V_{R}\left(C_{1}\right)$ consists of a small vertex of $K^{\prime}$. Once we have found a good $K^{\prime}$, we can use the Embedding lemma to find at most $|H|-1$ vertex-disjoint copies of $H$ in $G$ such that their removal from $G$ ensures that $\left|V_{G}\left(C_{1}\right)\right|$ is divisible by $|H|$, as desired. (In case (i) precisely one vertex in each of these copies of $H$ lies in $V_{G}\left(C_{1}\right)$ while in case (ii) precisely $|H|-1$ vertices in each of these copies of $H$ lies in $V_{G}\left(C_{1}\right)$.) So it suffices to find a good copy of $K_{r+1}^{-}$.

Let $S$ denote the set of neighbours of $V_{1}$ outside $V_{R}\left(C_{1}\right)$ in $R$. Let $K$ be the set of vertices $V \in S$ with $d_{R}(V)<\left(1-1 / \chi_{c r}(H)+\eta / 4\right)|R|$. By (13), $K$ induces a clique in $R$. If $|K| \geq r$, then we have a found a good copy of $K_{r+1}^{-}$(consisting of $V_{1}$ and $r$ vertices of $K)$. So we may assume that $|K|<r$.

Since $r \geq 3$ we have that $d_{R}\left(V_{1}\right) \geq(1 / 2+\eta / 4)|R|$. So $|S \backslash K| \geq \eta|R| / 4-r>0$. Thus we can choose $V_{2} \in S \backslash K$. By (11) the number of common neighbours of $V_{1}$ and $V_{2}$ in $R$ is at least

$$
\left(1-\frac{2}{r-1+\gamma}+\frac{\eta}{4}\right)|R|
$$

We first consider the case when at least $\left(1-\frac{2}{r-1+\gamma}+\frac{\eta}{4}\right)\left|V(R) \backslash V_{R}\left(C_{1}\right)\right|$ common neighbours of $V_{1}$ and $V_{2}$ lie outside $V_{R}\left(C_{1}\right)$. We claim that we can find $V_{3}, \ldots, V_{r} \in S \backslash K$ which form a $K_{r}$ with $V_{1}$ and $V_{2}$. Suppose that we have found $V_{3}, \ldots, V_{i}$ where $2 \leq i \leq r-1$. Note that Claim 20 and the definition of $S$ imply that for $j \geq 2$ the number of neighbours of $V_{j}$ outside $V_{R}\left(C_{1}\right)$ is at least $(1-1 /(r-1+\gamma))\left|V(R) \backslash V_{R}\left(C_{1}\right)\right|$. Together with (15), this implies that the common neighbourhood of $V_{1}, \ldots, V_{i}$ outside $V_{R}\left(C_{1}\right)$ has size at least

$$
\left(1-\frac{i}{r-1+\gamma}+\frac{\eta}{4}\right)\left|V(R) \backslash V_{R}\left(C_{1}\right)\right| \geq \frac{\eta}{4}\left|V(R) \backslash V_{R}\left(C_{1}\right)\right|>r>|K| .
$$

This shows that we can find $V_{i+1}$ and more generally $V_{3}, \ldots, V_{r}$ as required. A similar calculation as in (16), shows that the common neighbourhood of $V_{2}, \ldots, V_{r}$ outside $V_{R}\left(C_{1}\right)$ is non-empty and so contains some vertex $V_{r+1}$ say. Together with $V_{1}, \ldots, V_{r}$, $V_{r+1}$ forms a good copy of $K_{r+1}^{-}$.

Now consider the case when at least $\left(1-\frac{2}{r-1+\gamma}+\frac{\eta}{4}\right)\left|V_{R}\left(C_{1}\right)\right|$ common neighbours of $V_{1}$ and $V_{2}$ lie inside $V_{R}\left(C_{1}\right)$. Since $\eta\left|V_{R}\left(C_{1}\right)\right| / 4 \geq \eta\left|B^{*}\right| / 4 \geq r$ we can argue as in the previous case. Indeed, this time we choose $V_{3}, \ldots, V_{r}$ inside $V_{R}\left(C_{1}\right)$ to obtain a copy of $K_{r}$ in $R$ with one vertex, namely $V_{2}$, outside $V_{R}\left(C_{1}\right)$. We also choose a vertex $V_{r+1}$ 
inside $V_{R}\left(C_{1}\right)$ that is adjacent to $V_{1}, V_{3}, \ldots, V_{r}$. Again, $V_{1}, \ldots, V_{r+1}$ form a good copy of $K_{r+1}^{-}$.

Case 2. Every component $C \in \mathcal{C}$ with $\left|V_{R}(C)\right| \leq|R| / 2$ is such that $d_{R}(V)<(1-$ $\left.1 / \chi_{c r}(H)+\eta / 4\right)|R|$ for all $V \in V_{R}(C)$.

Together with (13) this implies that $V_{1} V_{2} \in E(R)$ for all $V_{1} \in V_{R}\left(C_{1}\right), V_{2} \in V_{R}\left(C_{2}\right)$ where $C_{1}, C_{2} \in \mathcal{C}$ are such that $\left|V_{R}\left(C_{1}\right)\right|,\left|V_{R}\left(C_{2}\right)\right| \leq|R| / 2$. But this means that there is only one component $C^{\prime} \in \mathcal{C}$ with $\left|V_{R}\left(C^{\prime}\right)\right| \leq|R| / 2$. So $F$ consists of precisely two components $C^{\prime}$ and $C^{\prime \prime}$ where $V_{R}\left(C^{\prime}\right)$ forms a clique in $R$ and $\left|V_{R}\left(C^{\prime \prime}\right)\right|>|R| / 2$.

We first consider the case when $r=3$. Note that $R$ contains an edge between $V_{R}\left(C^{\prime}\right)$ and $V_{R}\left(C^{\prime \prime}\right)$. Indeed, if not then for any $V^{\prime} \in V_{R}\left(C^{\prime}\right)$ and $V^{\prime \prime} \in V_{R}\left(C^{\prime \prime}\right)$ by (13) we have that $d_{R}\left(V^{\prime}\right)+d_{R}\left(V^{\prime \prime}\right) \geq 2\left(1-1 / \chi_{c r}(H)+\eta / 4\right)|R|>|R|$ and so there must be an edge from $V^{\prime}$ to $V_{R}\left(C^{\prime \prime}\right)$ or from $V^{\prime \prime}$ to $V_{R}\left(C^{\prime}\right)$, a contradiction.

So since $\left|V_{R}\left(C^{\prime}\right)\right| \geq\left|B^{*}\right| \geq r+m$ we have a copy $K_{r+m}^{\prime}$ of $K_{r+m}$ in $V_{R}\left(C^{\prime}\right)$ such that there is a cluster $V^{\prime \prime} \in V_{R}\left(C^{\prime \prime}\right)$ adjacent to one of the clusters, $V^{\prime}$ say, of $K_{r+m}^{\prime}$. Using the definition of $m$ and the Embedding lemma we can find at most $|H|-1$ copies of $H$ in $G$ each containing precisely one vertex in $V_{G}\left(C^{\prime \prime}\right)$ such that their removal ensures that $|H|$ divides $\left|V_{R}\left(C^{\prime}\right)\right|$ and thus also $\left|V_{R}\left(C^{\prime \prime}\right)\right|$. (Indeed, by definition of $m$ there exists a vertex $y$ of $H$ such that $\chi(H[N(y)])=r-2=1$ and such that some 1-colouring of $N(y)$ can be extended to an $(r+m)$-colouring of $H$. So in our copies of $H$ the vertex $y$ will lie in $V^{\prime \prime}, N(y)$ will lie in $V^{\prime}$ and the remaining vertices of $H$ will lie in $V\left(K_{r+m}^{\prime}\right)$.)

Now suppose that $r \geq 4$. We claim that there exists $V^{\prime \prime} \in V_{R}\left(C^{\prime \prime}\right)$ which sends at least $r$ edges to $V_{R}\left(C^{\prime}\right)$ in $R$. Suppose not. Then no $V \in V_{R}\left(C^{\prime \prime}\right)$ is joined to all of $V_{R}\left(C^{\prime}\right)$. Together with the definition of $C^{\prime}$ and (13) this implies that $d_{R}(V) \geq$ $\left(1-1 / \chi_{c r}(H)+\eta / 4\right)|R|$. But then $\left|V_{R}\left(C^{\prime}\right)\right|<|R| / \chi_{c r}(H)$ since otherwise $V$ is joined to $\eta|R| / 4 \geq r$ vertices in $V_{R}\left(C^{\prime}\right)$. By assumption there are less than $r\left|V_{R}\left(C^{\prime \prime}\right)\right|<r|R|$ edges between $V_{R}\left(C^{\prime}\right)$ and $V_{R}\left(C^{\prime \prime}\right)$ in $R$. Moreover, by (13) and since $\left|V_{R}\left(C^{\prime}\right)\right|<|R| / \chi_{c r}(H)$ every cluster in $V_{R}\left(C^{\prime}\right)$ sends at least $\left(1-3 / \chi_{c r}(H)+\eta / 4\right)|R|>\eta|R| / 4$ edges to $V_{R}\left(C^{\prime \prime}\right)$. So $\eta|R|\left|V_{R}\left(C^{\prime}\right)\right| / 4<r|R|$. But $\left|V_{R}\left(C^{\prime}\right)\right| \geq\left|B^{*}\right| \geq 4 r / \eta$ by definition of $B^{*}$ and so $\eta|R|\left|V_{R}\left(C^{\prime}\right)\right| / 4 \geq r|R|$, a contradiction. So indeed there exists a vertex $V^{\prime \prime} \in V_{R}\left(C^{\prime \prime}\right)$ sending at least $r$ edges to $V_{R}\left(C^{\prime}\right)$. As before, we can remove at most $|H|-1$ copies of $H$ from $G$ to ensure that $|H|$ divides both $\left|V_{R}\left(C^{\prime}\right)\right|$ and $\left|V_{R}\left(C^{\prime \prime}\right)\right|$.

Claim 22. We can make $\left|V_{G}(B)\right|$ divisible by $|H|$ for all $B \in \mathcal{B}^{*}$ by removing at most $\left|\mathcal{B}^{*}\right||H|$ copies of $H$ from $G$.

Proof. Our first aim is to take out some copies of $H$ in $G$ to achieve that $\left|V_{G}(C)\right|$ is divisible by $|H|$ for each $C \in \mathcal{C}$. We apply Claim 21 to remove at most $|H|-1$ copies of $H$ from $G$ to ensure that $\left|V_{G}\left(C_{1}\right)\right|$ is divisible by $|H|$ for some component $C_{1} \in \mathcal{C}$ with $\left|V_{R}\left(C_{1}\right)\right| \leq|R| / 2$. Next we consider the graphs $F_{1}:=F-V\left(C_{1}\right)$ and $R_{1}:=R-V_{R}\left(C_{1}\right)$ instead of $F$ and $R$. Claim 20 and (13) together imply that

$$
d_{R_{1}}\left(V_{j_{1}}\right)+d_{R_{1}}\left(V_{j_{2}}\right) \geq 2\left(1-\frac{1}{r-1+\gamma}+\frac{\eta}{4}\right)\left|R_{1}\right|
$$

for all $V_{j_{1}} \neq V_{j_{2}} \in V\left(R_{1}\right)$ with $V_{j_{1}} V_{j_{2}} \notin E\left(R_{1}\right)$. Now suppose that $|\mathcal{C}| \geq 3$. Then similarly as in the proof of Claim 21 we can find a component $C_{2} \in \mathcal{C}$ with $\left|V_{R}\left(C_{2}\right)\right| \leq$ 
$\left|R_{1}\right| / 2$ and such that by removing at most $|H|-1$ copies of $H$ from $G$ we ensure that $|H|$ divides $\left|V_{G}\left(C_{2}\right)\right|$. As $|G|$ was divisible by $|H|$ we can continue in this fashion to achieve that $\left|V_{G}(C)\right|$ is divisible by $|H|$ for each $C \in \mathcal{C}$.

During this process we have to take out at most $(|\mathcal{C}|-1)(|H|-1)$ copies of $H$ in $G$. Now consider each $C \in \mathcal{C}$ separately. By proceeding as in the connected case for each $C$ and taking out at most $(|C|-1)(|H|-1)$ further copies of $H$ in each case, we can make $\left|V_{G}(B)\right|$ divisible by $|H|$ for all $B \in \mathcal{B}^{*}$. Hence, in total we have taken out at most $(|\mathcal{C}|-1)(|H|-1)+\left(\left|\mathcal{B}^{*}\right|-|\mathcal{C}|\right)(|H|-1) \leq\left|\mathcal{B}^{*}\right||H|$ copies of $H$. (Note that $\left|\mathcal{B}^{*}\right||H|$ is also an upper bound on the number of copies of $H$ removed from $G$ in the case when $r=2$.)

7.5. Applying the Blow-up lemma. We now consider all the copies $B_{1}^{\prime}, \ldots, B_{\ell^{\prime}}^{\prime}$ of $B^{\prime}$ in the $B^{\prime}$-packing of $R$, where the vertices of $R$ are the modified clusters (i.e. they do not contain the vertices contained in the copies of $H$ removed in Sections 7.3 and 7.4). For each $i \leq \ell^{\prime}$ let $G_{i}^{\prime}$ denote the $r$-partite subgraph of $G^{\prime}$ whose $j$ th vertex class is the union of all the clusters lying in the $j$ th vertex class of $B_{i}^{\prime}($ for $j=1, \ldots, r$ ). In Section 7.4 we made $\left|G_{i}^{\prime}\right|=\left|V_{G}\left(B_{i}^{\prime}\right)\right|$ divisible by $|H|$ for each $i$. Moreover, in Section 7.3 we removed at most $\beta L^{\prime}$ vertices from each cluster. In Section 7.4 we removed only a bounded number of further vertices. So altogether we removed at most $2 \beta L^{\prime}$ vertices from each cluster. Since $\beta \ll \lambda \ll \gamma, 1-\gamma$ we may apply Lemma 14 to conclude that the complete $r$-partite graph whose vertex classes are the same as the vertex classes of $G_{i}^{\prime}$ has a perfect $H$-packing.

We observed at the end of Section 7.2 that the choice of those copies of $H$ removed in Section 7.3 ensures that all the bipartite subgraphs corresponding to edges of $B_{i}^{\prime}$ are still $(5 \varepsilon, d / 5)$-super-regular. In Section 7.4 we only removed a bounded number of further vertices from each cluster. So after Section 7.4 the bipartite subgraphs of $G_{i}^{\prime}$ are still

$(6 \varepsilon, d / 6)$-super-regular. Hence, for each $i=1, \ldots, \ell^{\prime}$, we may apply the Blow-up lemma to find a perfect $H$-packing in $G_{i}^{\prime}$. All these $H$-packings together with the copies of $H$ chosen previously form a perfect $H$-packing in $G$, as desired.

\section{REFERENCES}

[1] N. Alon and R. Yuster, H-factors in dense graphs, J. Combin. Theory B 66 (1996), 269-282.

[2] R. Diestel, Graph Theory, Graduate texts in Mathematics 173 (3rd Edition), Springer-Verlag 2005.

[3] A. Hajnal and E. Szemerédi, Proof of a conjecture of Erdős, Combinatorial Theory and its Applications vol. II 4 (1970), 601-623.

[4] P. Hell and D.G. Kirkpatrick, On the complexity of general graph factor problems, SIAM J. Computing 12 (1983), 601-609.

[5] K. Kawarabayashi, $K_{4}^{-}$-factor in a graph, J. Graph Theory 39 (2002), 111-128.

[6] H.A. Kierstead and A.V. Kostochka, An Ore-type Theorem on Equitable Coloring, J. Combin. Theory B 98 (2008), 226-234.

[7] H.A. Kierstead, A.V. Kostochka and G. Yu, Extremal graph packing problems: Ore-type versus Dirac-type, Surveys in Combinatorics, to appear.

[8] J. Komlós, The Blow-up Lemma, Combinatorics, Probability and Computing 8 (1999), 161-176.

[9] J. Komlós, Tiling Turán Theorems, Combinatorica 20 (2000), 203-218.

[10] J. Komlós, G.N. Sárközy and E. Szemerédi, Blow-up Lemma, Combinatorica 17 (1997), 109-123.

[11] J. Komlós, G.N. Sárközy and E. Szemerédi, Proof of the Alon-Yuster conjecture, Discrete Math. 235 (2001), 255-269. 
[12] J. Komlós and M. Simonovits, Szemerédi's Regularity Lemma and its applications in graph theory, Combinatorics: Paul Erdös is eighty, Vol.2 (1996), 295-352.

[13] D. Kühn and D. Osthus, Critical chromatic number and the complexity of perfect packings in graphs, 17th ACM-SIAM Symposium on Discrete Algorithms (SODA 2006), 851-859.

[14] D. Kühn and D. Osthus, The minimum degree threshold for perfect graph packings, Combinatorica 29 (2009), 65-107.

[15] D. Kühn and D. Osthus, Embedding large subgraphs into dense graphs, Surveys in Combinatorics, to appear.

[16] O. Ore, Note on Hamilton circuits, Amer. Math. Monthly 67 (1960), 55.

[17] A. Shoukoufandeh and Y. Zhao, Proof of a conjecture of Komlós, Random Struct. Alg. 23 (2003), 180-205.

[18] E. Szemerédi, Regular partitions of graphs, Problémes Combinatoires et Théorie des Graphes Colloques Internationaux CNRS 260 (1978), 399-401.

[19] A. Treglown, The Regularity Lemma and applications to packings in graphs, MSci thesis, University of Birmingham, 2007.

[20] A. Treglown, PhD thesis, University of Birmingham, in preparation.

[21] R. Yuster, Combinatorial and computational aspects of graph packing and graph decomposition, Computer Science Review 1 (2007), 12-26.

Daniela Kühn, Deryk Osthus \& Andrew Treglown

School of Mathematics

University of Birmingham

Edgbaston

Birmingham

B15 2TT

UK

E-mail addresses: $\{$ kuehn,osthus, treglowa\}@maths.bham.ac.uk 